\title{
Interstellar Hydroxyl Masers in the Galaxy. II. Zeeman Pairs and the Galactic Magnetic Field
}

\author{
Vincent L. Fish, Mark J. Reid and Alice L. Argon \\ Harvard-Smithsonian Center for Astrophysics \\ 60 Garden Street, Cambridge, MA 02138 \\ vfish@cfa.harvard.edu, mreid@cfa.harvard.edu, aargon@cfa.harvard.edu \\ and \\ Karl M. Menten \\ Max-Planck-Institut für Radioastronomie \\ Auf dem Hügel 69, Bonn, D-53121 Germany \\ kmenten@mpifr-bonn.mpg.de
}

\begin{abstract}
We have identified and classified Zeeman pairs in the survey by Argon, Reid, \& Menten of massive star-forming regions with $18 \mathrm{~cm}\left({ }^{2} \Pi_{3 / 2}, J=3 / 2\right) \mathrm{OH}$ maser emission. We have found a total of more than 100 Zeeman pairs in more than 50 massive star-forming regions. The magnetic field deduced from the Zeeman splitting has allowed us to assign an overall line-of-sight magnetic field direction to many of the massive star-forming regions. Combining these data with other data sets obtained from $\mathrm{OH}$ Zeeman splitting, we have looked for correlations of magnetic field directions between star-forming regions scattered throughout the Galaxy. Our data do not support a uniform, Galactic-scale field direction, nor do we find any strong evidence of magnetic field correlations within spiral arms. However, our data suggest that in the Solar neighborhood the magnetic field outside the Solar circle is oriented clockwise as viewed from the North Galactic Pole, while inside the Solar circle it is oriented counterclockwise. This pattern, including the magnetic field reversal near the Sun, is in agreement with results obtained from pulsar rotation measures.
\end{abstract}

Subject headings: masers — ISM: magnetic fields — Galaxy: disk — H II regions — radio lines: ISM 


\section{Introduction}

Hydroxyl $(\mathrm{OH})$ masers are an excellent tool for probing the environment of massive starforming regions (SFRs). They are bright and easily observable and act as point tracers of the local velocity and magnetic fields, owing to their large Zeeman splitting coefficient. For the groundstate ${ }^{2} \Pi_{3 / 2}, J=3 / 2$ transitions, the Zeeman splitting coefficient is as large as $0.59 \mathrm{~km} \mathrm{~s}^{-1} \mathrm{mG}^{-1}$. For a typical maser linewidth of $0.3 \mathrm{~km} \mathrm{~s}^{-1}$, a milligauss-strength magnetic field is sufficient to cleanly separate Zeeman components of the $1665 \mathrm{MHz}$ transition, and even fields as weak as several hundred microgauss can be detected. In addition, the sense of the splitting indicates the projected line-of-sight magnetic field orientation.

Davies (1974) first hypothesized that a connection exists between magnetic field directions obtained from molecular Zeeman splitting and a large-scale Galactic magnetic field. He found that eight $\mathrm{OH}$ maser sources (and several H I clouds) mostly near the Solar circle with observable Zeeman splitting displayed magnetic fields aligned in the clockwise direction when viewed from the North Galactic Pole. Reid \& Silverstein (1990) conducted a literature search and found a total of 17 reliable $\mathrm{OH}$ maser sources with detectable Zeeman pairs in the ${ }^{2} \Pi_{3 / 2}, J=3 / 2$ transitions. Of these 17, 14 had line-of-sight field directions consistent with an overall clockwise Galactic field. In the largest survey to date, Baudry et al. (1997) extended this dataset to include a total of 46 sources with Zeeman pairs in the ${ }^{2} \Pi_{3 / 2}, J=3 / 2$ and $J=5 / 2$ transitions of $\mathrm{OH}$. Excluding the Galactic center, they found 28 sources consistent with a clockwise magnetic field and 17 sources consistent with a counterclockwise field.

Argon, Reid, \& Menten (2000, henceforth Paper I) conducted a survey of 91 interstellar regions with known ${ }^{2} \Pi_{3 / 2}, J=3 / 2 \mathrm{OH}$ maser emission. They identified and mapped maser spots in the regions. In this paper we extend the analysis of Paper I by identifying Zeeman pairs toward massive SFRs, many of which contain ultracompact (UC) H II regions. We also investigate whether or not the magnetic fields deduced from $\mathrm{OH}$ Zeeman splitting around massive SFRs are related to the large-scale magnetic field of the Galaxy.

\section{Observations}

A total of 91 maser sources were observed by Paper I with the A configuration of the National Radio Astronomy Observatory's Very Large Array ${ }^{1}$ in Socorro, NM. All sources were observed in both left- and right-circular polarization in at least one main-line ${ }^{2} \Pi_{3 / 2}, J=3 / 218 \mathrm{~cm} \mathrm{OH}$ transition (1665.4018 and $1667.3590 \mathrm{MHz}$ ), and some were also observed in one or both of the satellite-line transitions (1612.2310 and $1720.5300 \mathrm{MHz}$ ). The $0.763 \mathrm{kHz}$ spectral channel separation used corresponds to a velocity separation of $0.14 \mathrm{~km} \mathrm{~s}^{-1}$ for the main-line transitions (FWHM

\footnotetext{
${ }^{1}$ The National Radio Astronomy Observatory is a facility of the National Science Foundation operated under cooperative agreement by Associated Universities, Inc.
} 
$\sim 20 \%$ larger). Further details of the observations can be found in Paper I.

\section{Zeeman Pair Selection Criteria}

In the presence of a magnetic field, the degeneracy of the magnetic sublevels of the $\mathrm{OH}$ molecule is broken. For any given hyperfine transition, this results in three or more spectral lines with differing polarization properties — the Zeeman effect (see Davies 1974, for more details). OH masers often display pairs of strong left and right elliptically polarized $\sigma$-components. For reasons that are not well understood, purely linearly polarized $\pi$-components are rarely, if ever, detected.

The task of identifiying Zeeman pairs - two oppositely elliptically polarized lines at the same position on the sky - is relatively simple, provided one has sufficient angular resolution to resolve maser clusters (e.g., Reid et al. 1980). However, with the VLA one can only achieve an angular resolution of $\approx 1$ arcsec for the $\mathrm{OH}$ transitions at wavelengths of $18 \mathrm{~cm}$. Were the emission in any spectral channel to come from only one (unresolved) spot on the sky, this would not be a significant limitation. However, in reality the emission in any given spectral channel can come from spectral blends of masers at different positions in the source separated by less than the beam size. Thus, great care must be taken to determine Zeeman pairs from the Paper I data.

In order to identify Zeeman pairs, a modified version of the NRAO Astronomical Image Processing System (AIPS) task JMFIT was used to identify the brightest maser emission spot in each spectral channel (velocity plane) for any given source. A maser line was identified when emission was seen from approximately the same position in at least three adjacent velocity channels, with the strongest emission not found on the edge channels. This resulted in a large data set that could be automatically searched for Zeeman pairs.

Ideally, one would like to find Zeeman pairs in which the oppositely circularly polarized components were coincident on the sky to better than their apparent spot sizes. However, Reid et al. (1980) showed that $\mathrm{OH}$ masers in $\mathrm{W} 3(\mathrm{OH})$ clustered on a scale of $3 \times 10^{15} \mathrm{~cm}\left(0^{\prime \prime} .09\right.$ at $2.2 \mathrm{kpc}$; distances are discussed in $\S 4.1$ and listed in Table 1) and that oppositely circularly polarized maser lines within a cluster gave reliable magnetic field measurements when interpreted as Zeeman pairs. Thus, we searched for oppositely circularly polarized maser lines with projected positions separated by less than $3 \times 10^{15} \mathrm{~cm}$. While the main-line $1.6 \mathrm{GHz} \sigma$-components are in general elliptically polarized, it is almost functionally equivalent to treat them as being circularly polarized for the purposes of right-circular polarization (RCP) and left-circular polarization (LCP) VLA data. The caveat is that, for example, a right-elliptically polarized maser component, being the sum of circularly and linearly polarized emission, may appear in both RCP and LCP data at the same velocity, although the LCP emission will be much weaker, as the LCP feed is sensitive to only half of the (presumed small) linear component of the full elliptical polarization.

Some Zeeman pairs consisted of single RCP and LCP lines that were coincident on the sky to better than $3 \times 10^{15} \mathrm{~cm}$. We consider these easily recognizable and reliable Zeeman pairs. 
However, more than one maser line in the same polarization would often be seen within the assumed $3 \times 10^{15} \mathrm{~cm}$ clustering length, such that it was unclear which should be paired with the maser spot in the other polarization. While such data are insufficient to make an unambiguous Zeeman pair identification in all such cases, sometimes corroborating evidence from another maser transition (or occasionally even the same transition) in the same spatial region allowed the identification of Zeeman pairs. In other cases, the line-of-sight field direction was deducible, even if unambiguous identification of a Zeeman pair was not possible, for example, when multiple LCP and RCP lines were seen, but each RCP line was at a higher velocity than any of the LCP lines. We felt it important to generate specific, objective, criteria that allowed an algorithmic approach to search for Zeeman pairs and accept or reject them without resorting to a visual examination of spectra on a case-by-case basis, which can lead to significant unquantifiable biases. Below we describe how we handled each of the complications.

For any given source, a "window" of radius $3 \times 10^{15} \mathrm{~cm}$ was centered on the strongest line in either polarization in a single transition. In principle, if the window included one line in each polarization, the lines would be identified as a Zeeman pair. However, emission channels key to identifying the second maser line in a Zeeman pair sometimes lay just outside the window when it was centered on the position of brightest emission, whereas were the window moved between the position of the lines, it would pass the $<3 \times 10^{15} \mathrm{~cm}$ separation criterion. To overcome this problem, the window center was moved from the centroid of strongest emission along a grid, in increments of $5 \%$ of the window radius, out to a maximum displacement of $50 \%$ of the radius in both the R.A. and Dec directions. The window containing the largest number of lines was used, provided that at least one line of each polarization was included. "Tiebreaker" criteria consisted first of choosing the window with the largest number of velocity channels represented and second of choosing the window whose offset from the grid center was smallest. Next, all velocity channels contained in the chosen window were flagged as used, and the process was iterated with the remaining maser spots. In this way, the entire source was partitioned into windows consisting of potential Zeeman pairs. This partitioning was done independently for each maser transition for which data were collected for the source.

Identification of Zeeman pairs in windows containing only one maser line in each polarization is straightforward. Figure 1a shows spectra from two windows for G351.775-0.538, one in each of the 1665 and $1667 \mathrm{MHz}$ transitions. The $1665 \mathrm{MHz}$ window contains two oppositely circularly-polarized maser lines. Removing the effects of the Zeeman splitting from a magnetic field of $-3.80 \pm 0.03 \mathrm{mG}$ (i.e., oriented in the hemisphere toward the Sun.), the center velocity of the emission would be at $-26.74 \pm 0.02 \mathrm{~km} \mathrm{~s}^{-1}$. The $1667 \mathrm{MHz}$ window, centered 120 mas away, shows a similar splitting of $-3.66 \pm 0.06 \mathrm{mG}$ centered at a velocity of $-26.79 \pm 0.02 \mathrm{~km} \mathrm{~s}^{-1}$. While a large fraction of the Zeeman pairs we identified were found in windows containing exactly one maser line in each polarization, a few of these pairs appeared with a corroborating nearby pair of a similar magnetic field in another transition.

Table 1 is a list of Zeeman pairs with "non-zero" velocity separation. The flux, velocity, and 
velocity width of lines was determined by a Gaussian fit to the center 5 channels of each line when available, else to 4 or only 3 channels. (By our criteria for a maser line, the center 3 channels must necessarily be detected.) Pairs are only included in this table if the velocity difference between the LCP and RCP lines exceeds twice the signal-to-noise-limited velocity error, taken to be the sum in quadrature of the HWHM line width divided by the signal-to-noise ratio of each of the lines. However, in no case is a pair of lines allowed to satisfy this criterion if the velocity center separation is less than $5 \%$ of the quadrature sum of the line widths, or typically about $0.02 \mathrm{~km} \mathrm{~s}^{-1}$. These "pairs" may be actual Zeeman pairs with a small splitting due to a small magnetic field, or they may be single maser spots with a large linear polarization fraction, but the limited spatial and velocity resolution of the VLA and the absence of full-polarization observations render it difficult to decide between these two cases. These "near zero" velocity separation maser spots are listed in Table 2.

While identification of many of the pairs listed in Table 1 was straightforward, not all windows were so simple to interpret. When a window contains more than one maser line in a polarization, interpreting the Zeeman pattern is more complicated. In this case, it may still be possible to know the light-of-sight direction of the magnetic field, but not its strength. Figure 1b shows the spectrum of one window associated with G032.744-0.076. There is one RCP maser line (labelled 1) centered at $32.38 \mathrm{~km} \mathrm{~s}^{-1}$ and two LCP lines (2 and 3) at higher velocities (34.60 and $35.02 \mathrm{~km} \mathrm{~s}^{-1}$, respectively). Additionally, there is one feature (4) that does not meet our criteria as a maser line, since the low-velocity side of the line is not seen, probably owing to blending with a stronger line elsewhere in the source. It is unclear which LCP line should be associated with the RCP line as a Zeeman pair. Regardless of the exact pairing, the RCP line is at lower velocity than any LCP line, so the line-of-sight orientation of the magnetic field is known to be pointed toward the Sun. Cases such as this, where the field direction is known but its strength is not, are frequent among our data. When a range of values is quoted for the magnetic field in Table 1, the direction of the magnetic field is well-determined, and the smallest and largest possible magnetic field intensities are given. Some line parameters not listed in the table are given in the notes on individual sources.

Sometimes when a window contains enough maser lines such that the magnetic field strength or direction is ambiguous, supporting evidence may exist to suggest one pairing above all others. If a pairing in one transition implies a magnetic field strength and direction consistent with that of a pair in another transition at nearly the same location, that pairing is accepted in preference to all other possible pairings. Figure 1c shows a $1665 \mathrm{MHz}$ and a $1667 \mathrm{MHz}$ window in K3-50, coincident to within 46 mas. In each window, there is one RCP line (1) at lower velocity than either of the two LCP lines (2 and 3), so the line-of-sight field is oriented toward the Sun. But the field magnitude can also be deduced, assuming that the nearly overlapping windows in the two transitions are measuring the field at approximately the same location. In the $1665 \mathrm{MHz}$ window, pairing lines 1 and 2 would give a magnetic field of $-1.5 \mathrm{mG}$, while the $1-3$ pairing implies a $-2.5 \mathrm{mG}$ field. In the $1667 \mathrm{MHz}$ window, the 1-2 pairing yields $-0.8 \mathrm{mG}$, while $1-3$ yields $-2.6 \mathrm{mG}$. The close agreement between the field values for the 1-3 pairs for the two transitions suggests that 
these Zeeman pairs should be favored over the other pairing possibilities. For definiteness, when appealing to corroborating evidence from one transition to resolve pairing ambiguities in another transition, we required $\Delta B \leq 0.4 \mathrm{mG}, \Delta v_{c} \leq 0.2 \mathrm{~km} \mathrm{~s}^{-1}$, and $\Delta \theta \leq 0^{\prime \prime} .2 \sec z \tan z$, where $\Delta B$ is the difference of the implied magnetic field strengths, $\Delta v_{c}$ is the separation of the center velocity of each pair, $\Delta \theta$ is the positional offset between pairs, and $z$ is the zenith angle of the source as viewed from the location of the VLA at transit. As noted in Paper I, relative positional offsets between different $\mathrm{OH}$ transition frequencies are $\approx 0^{\prime \prime} .1$ for high-declination sources, but grow to several arcseconds for sources at very low declination $\left(\lesssim-40^{\circ}\right)$. The function "sec $z \tan z$ " was chosen in the spirit of the plane-parallel approximation for the atmospheric contribution to the relative error between observations of different transitions (see Reid et al. 1999).

If two pairings in the same window in the same transition imply the same magnetic field direction and strength, those pairings are also accepted over all other pairings. This situation arises when two Zeeman pairs in the same transition are encompassed in the same window. Figure 1d shows a $1665 \mathrm{MHz}$ window for G45.465+0.047 that contains a cluster of maser lines. Since all of the RCP lines are at a higher velocity than the LCP lines, the magnetic field is oriented away from the Sun. However, the velocities associated with the line centers favor two particular pairings: pairing lines 1 and 4 yields a magnetic field strength of $3.7 \mathrm{mG}$, and 2 and 5 yield $3.8 \mathrm{mG}$.

Occasionally, a window would produce a spectrum with a clear, strong line in one polarization and an incomplete line in the opposite polarization. A closer look at the data would show that one or two critical points of the incomplete line lay just outside the window. By increasing the size of the window, a Zeeman pair was often identified. Using a larger window (generally twice the clustering radius) can be justified for two reasons. First, the window radius of $3 \times 10^{15} \mathrm{~cm}$ is based on the clustering scale analysis of Reid et al. (1980), which is itself based on only one source: W3 $(\mathrm{OH})$, assuming the photometric distance of $2.2 \mathrm{kpc}$ (Humphreys 1978). The kinematic distance of $4.3 \mathrm{kpc}$, assuming the $\mathrm{NH}_{3}$ LSR velocity of $-45 \mathrm{~km} \mathrm{~s}^{-1}$ (Reid, Myers, \& Bieging 1987) and a flat $R_{0}=8.5 \mathrm{kpc}, \Theta(R)=220 \mathrm{~km} \mathrm{~s}^{-1}$ rotation curve, would correspond to a clustering scale of $6 \times 10^{15} \mathrm{~cm}$, or twice that which is derived from the photometric distance. In any case, it is unclear to what extent this varies among massive SFRs. Second, the distance to some of the massive SFRs is unknown. If the assumed distance to the source is greater than the actual distance, the (angular) window radius used would be proportionally smaller than the desired $3 \times 10^{15} \mathrm{~cm}$. For sources for which the kinematic distance ambiguity has not been resolved, the Zeeman pair identification analysis was done twice, assuming each of the near and far distances for calculation of the clustering scale. Zeeman pairs identified assuming either distance were accepted and are listed in Table 1. Despite the distance uncertainty, we believe that Zeeman pairs identified around these sources are still reliable indicators of the local magnetic field. When the far kinematic distance is more than a few times the near kinematic distance, possible errors involve partitioning a far source into windows that are too large or a near source into windows that are too small. In the former case, nearly all of the maser emission of a source (presumedly) located at the far kinematic distance would be concentrated in a single window when the near distance was used for the angular 
clustering scale, resulting in confusion of Zeeman pairs. In the latter case, a (presumed) near source would be micropartitioned into windows of small radius, rejecting many Zeeman pairs coincident to within the desired linear clustering scale. Thus, misresolving the near/far kinematic distance ambiguity results in the identification of fewer Zeeman pairs, regardless of whether a far source is assumed to be near or a near source is assumed to be far.

Zeeman pairs were also graded to reflect the uncertainty in pairing. Pairs from windows containing only one line in each polarization were assigned a grade of B, unless they were confirmed by a similar pair in a second transition, in which case they were given a grade of A. All pairs whose pairing was ambiguous were given a grade of $\mathrm{C}$, even if the ambiguity was resolved by appealing to a second pair in the same or another transition. Pairs for which the separation of the Zeeman components exceeded the default window size were assessed a grade of $\mathrm{D}$. In one case in G081.871+0.781, a grade of D was assigned to a Zeeman pair believed to be spurious; for further details see $§ 4.3$. This grading system is in the spirit of, although not identical to, the system used in Reid \& Silverstein (1990), in which the main emphasis was on whether interferometry was used to identify Zeeman splitting, and if so, at what resolution. All of the Zeeman pairs listed in Table 1 were identified using interferometry. 
Table 1. Zeeman Pairs

\begin{tabular}{|c|c|c|c|c|c|c|c|c|c|c|c|c|}
\hline \multirow[b]{2}{*}{ Source } & \multirow[b]{2}{*}{ Alias } & \multirow[b]{2}{*}{$\begin{array}{l}\text { Dist. }^{a} \\
(\mathrm{kpc})\end{array}$} & \multirow[b]{2}{*}{$\begin{array}{l}\text { Freq. } \\
(\mathrm{MHz})\end{array}$} & \multirow{2}{*}{$\begin{array}{r}S^{\mathrm{b}} \\
(\mathrm{Jy})\end{array}$} & \multirow{2}{*}{$\begin{array}{c}\mathrm{LCP} \\
v_{\mathrm{LSR}}^{\mathrm{b}} \\
\left(\mathrm{km} \mathrm{s}^{-1}\right)\end{array}$} & \multirow[b]{2}{*}{$\begin{array}{c}\Delta v^{\mathrm{b}} \\
\left(\mathrm{km} \mathrm{s}^{-1}\right)\end{array}$} & \multirow[b]{2}{*}{$\begin{array}{r}S^{\mathrm{b}} \\
(\mathrm{Jy})\end{array}$} & \multirow{2}{*}{$\begin{array}{c}\mathrm{RCP}- \\
v_{\mathrm{LSR}}{ }^{\mathrm{b}} \\
\left(\mathrm{km} \mathrm{s}^{-1}\right)\end{array}$} & \multirow[b]{2}{*}{$\begin{array}{c}\Delta v^{\mathrm{b}} \\
\left(\mathrm{km} \mathrm{s}^{-1}\right)\end{array}$} & \multirow[b]{2}{*}{$\begin{array}{c}\Delta \theta^{\mathrm{c}} \\
(\operatorname{arcsec})\end{array}$} & \multirow[b]{2}{*}{$\begin{array}{c}B^{\mathrm{d}} \\
(\mathrm{mG})\end{array}$} & \multirow[b]{2}{*}{ Grade $^{\mathrm{e}}$} \\
\hline & & & & & & & & & & & & \\
\hline G000.547-0.852 & RCW 142 & 7.3 & 1667 & 0.23 & 8.96 & 0.65 & 3.72 & 6.33 & 0.43 & 0.046 & -7.4 & B \\
\hline G000.666-0.034 & Sgr B2M & 8.2 & 1720 & 6.84 & 61.09 & 0.28 & 14.21 & 61.25 & 0.32 & 0.028 & 0.7 & $\mathrm{~B}$ \\
\hline G000.670-0.058 & Sgr B2 & 8.2 & 1612 & 3.78 & 67.40 & 0.87 & 3.91 & 67.24 & 0.82 & 0.024 & -0.7 & $\mathrm{D}$ \\
\hline \multirow[t]{4}{*}{ G005.886-0.393 } & $\ldots$ & 3.8 & 1665 & 4.26 & 9.52 & 0.26 & 3.04 & 10.41 & 0.32 & 0.028 & 1.5 & $\mathrm{~A}$ \\
\hline & & & 1667 & 8.90 & 9.71 & 0.28 & 7.83 & 10.22 & 0.29 & 0.012 & 1.4 & $\mathrm{~A}$ \\
\hline & & & & 1.43 & 4.93 & 0.75 & 0.74 & 5.18 & 0.96 & 0.042 & 0.7 & B \\
\hline & & & & 1.07 & 6.10 & 0.80 & 1.50 & 7.08 & 0.56 & 0.080 & $0.2 \rightarrow 2.8$ & C \\
\hline \multirow[t]{3}{*}{ G009.622+0.195 } & $\cdots$ & 5.7 & 1665 & 2.41 & -1.07 & 0.42 & 8.06 & 1.75 & 0.37 & 0.018 & $3.9 \rightarrow 9.7$ & $\mathrm{C}$ \\
\hline & & & & 1.34 & 3.90 & 0.53 & 2.68 & 3.85 & 0.40 & 0.044 & $-0.1 \rightarrow-5.4$ & $\mathrm{C}$ \\
\hline & & & 1667 & 9.67 & 1.47 & 0.41 & 1.98 & 1.43 & 0.48 & 0.043 & -0.1 & $\mathrm{~B}$ \\
\hline \multirow[t]{2}{*}{ G010.624-0.385 } & $\cdots$ & 4.8 & 1667 & 43.03 & -0.59 & 0.35 & 44.13 & -1.98 & 0.25 & 0.045 & -3.9 & $\mathrm{~B}$ \\
\hline & & & & 0.80 & 2.08 & 0.30 & 1.26 & 0.10 & 0.33 & 0.049 & -5.6 & B \\
\hline G012.216-0.117 & $\ldots$ & 3.3 & 1665 & 15.26 & 29.07 & 0.49 & 12.32 & 27.55 & 0.50 & 0.042 & -2.6 & $\mathrm{~B}$ \\
\hline \multirow[t]{2}{*}{ G012.680-0.181 } & W33 B & 4.0 & 1665 & 20.29 & 64.45 & 0.50 & 4.45 & 63.34 & 0.43 & 0.035 & $-1.9 \rightarrow-6.3$ & $\mathrm{C}$ \\
\hline & & & 1667 & 5.45 & 64.50 & 0.48 & 3.62 & 62.50 & 0.62 & 0.029 & $-0.7 \rightarrow-7.5$ & $\mathrm{C}$ \\
\hline G012.908-0.259 & W33 A & 4.0 & 1667 & 69.34 & 39.93 & 0.49 & 5.12 & 40.41 & 0.75 & 0.023 & 1.4 & $\mathrm{~B}$ \\
\hline G017.639+0.155 & $\ldots$ & 2.1 & 1665 & 1.79 & 20.92 & 0.35 & 2.22 & 20.42 & 0.28 & 0.048 & -0.8 & $\mathrm{~B}$ \\
\hline \multirow[t]{2}{*}{ G020.081-0.135 } & $\ldots$ & 12.3 & 1665 & 0.66 & 42.90 & 0.48 & 2.22 & 48.64 & 0.39 & 0.060 & 9.7 & $\mathrm{~B}$ \\
\hline & & & & 10.99 & 46.60 & 0.39 & 1.42 & 49.75 & 0.48 & 0.016 & $5.3 \rightarrow 7.3$ & $\mathrm{C}$ \\
\hline G028.199-0.048 & $\ldots$ & 5.7 & 1665 & 11.48 & 94.92 & 0.36 & 19.23 & 94.89 & 0.27 & 0.008 & -0.1 & $\mathrm{~B}$ \\
\hline G031.412+0.307 & $\ldots$ & 6.2 & 1667 & 3.50 & 103.84 & 0.57 & 2.80 & 103.48 & 0.60 & 0.013 & -1.0 & B \\
\hline \multirow[t]{3}{*}{ G032.744-0.076 } & $\ldots$ & 2.3 & 1665 & 0.61 & 32.84 & 0.67 & 0.68 & 31.00 & 0.56 & 0.099 & -3.1 & B \\
\hline & & & & 1.04 & 35.02 & 0.58 & 1.73 & 32.38 & 0.46 & 0.059 & $-3.8 \rightarrow-4.5$ & $\mathrm{C}$ \\
\hline & & & & 0.39 & 36.44 & 0.37 & 0.92 & 39.58 & 0.35 & 0.053 & $5.3 \rightarrow 6.4$ & $\mathrm{C}$ \\
\hline \multirow[t]{3}{*}{ G034.257+0.154 } & $\cdots$ & 3.8 & 1665 & 16.04 & 58.14 & 0.37 & 79.02 & 58.22 & 0.38 & 0.015 & 0.1 & B \\
\hline & & & 1667 & 108.48 & 58.64 & 0.38 & 66.28 & 58.49 & 0.37 & 0.005 & -0.4 & B \\
\hline & & & & 7.28 & 57.50 & 0.32 & 10.52 & 57.41 & 0.28 & 0.006 & $-0.3 \rightarrow-1.6$ & $\mathrm{C}$ \\
\hline G035.024+0.350 & $\ldots$ & 3.1 & 1665 & 2.74 & 44.62 & 0.24 & 8.00 & 47.07 & 0.47 & 0.028 & $0.0 \rightarrow 5.5$ & $\mathrm{C}$ \\
\hline G035.197-0.743 & $\ldots$ & 2.2 & 1665 & 1.91 & 26.71 & 0.36 & 5.42 & 28.82 & 0.32 & 0.036 & $3.6 \rightarrow 4.2$ & $\mathrm{C}$ \\
\hline \multirow[t]{2}{*}{ G035.577-0.029 } & $\cdots$ & 10.5 & 1665 & 29.48 & 48.94 & 0.42 & 2.86 & 48.25 & 0.38 & 0.103 & -1.2 & $\mathrm{D}$ \\
\hline & & & & 6.87 & 50.79 & 0.33 & 57.64 & 49.01 & 0.28 & 0.116 & $-1.5 \rightarrow-4.9$ & $\mathrm{D}$ \\
\hline \multirow[t]{4}{*}{ G040.622-0.137 } & $\ldots$ & 2.1 & 1665 & 96.06 & 32.57 & 0.31 & 37.90 & 32.62 & 0.40 & 0.010 & 0.1 & B \\
\hline & & & & 1.31 & 34.47 & 0.34 & 2.14 & 35.74 & 0.32 & 0.020 & $0.5 \rightarrow 2.2$ & $\mathrm{C}$ \\
\hline & & & 1667 & 0.22 & 34.74 & 0.33 & 1.02 & 31.67 & 0.46 & 0.033 & -8.7 & $\mathrm{~B}$ \\
\hline & & & & 18.79 & 31.97 & 0.32 & 1.89 & 30.74 & 0.32 & 0.097 & $-0.3 \rightarrow-3.5$ & $\mathrm{C}$ \\
\hline
\end{tabular}


Table 1-Continued

\begin{tabular}{|c|c|c|c|c|c|c|c|c|c|c|c|c|}
\hline Source & Alias & $\begin{array}{l}\text { Dist. }^{a} \\
(\mathrm{kpc})\end{array}$ & $\begin{array}{l}\text { Freq. } \\
(\mathrm{MHz})\end{array}$ & $\begin{array}{r}S^{\mathrm{b}} \\
(\mathrm{Jy})\end{array}$ & $\begin{array}{c}\mathrm{LCP}- \\
v_{\mathrm{LSR}}{ }^{\mathrm{b}} \\
\left(\mathrm{km} \mathrm{s}^{-1}\right)\end{array}$ & $\begin{array}{c}\Delta v^{\mathrm{b}} \\
\left(\mathrm{km} \mathrm{s}^{-1}\right)\end{array}$ & $\begin{array}{r}S^{\mathrm{b}} \\
(\mathrm{Jy})\end{array}$ & $\begin{array}{c}\mathrm{RCP} \\
v_{\mathrm{LSR}}{ }^{\mathrm{b}} \\
\left(\mathrm{km} \mathrm{s}^{-1}\right)\end{array}$ & $\begin{array}{c}\Delta v^{\mathrm{b}} \\
\left(\mathrm{km} \mathrm{s}^{-1}\right)\end{array}$ & $\begin{array}{c}\Delta \theta^{\mathrm{c}} \\
(\operatorname{arcsec})\end{array}$ & $\begin{array}{c}B^{\mathrm{d}} \\
(\mathrm{mG})\end{array}$ & Grade $^{\mathrm{e}}$ \\
\hline G043.148+0.015 & W49 & 11.5 & 1612 & 2.30 & 13.20 & 0.26 & 2.59 & 13.88 & 0.44 & 0.037 & 2.9 & $\mathrm{D}$ \\
\hline \multirow[t]{3}{*}{ G043.165-0.028 } & W49 S & 11.5 & 1665 & 63.22 & 15.33 & 0.44 & 221.62 & 16.22 & 0.34 & 0.008 & 1.5 & B \\
\hline & & & & 45.65 & 19.11 & 0.36 & 6.60 & 22.55 & 0.52 & 0.016 & 5.8 & $\mathrm{~B}$ \\
\hline & & & & 26.02 & 13.23 & 1.06 & 35.52 & 13.68 & 0.57 & 0.013 & 0.8 & $\mathrm{~B}$ \\
\hline G043.796-0.127 & $\ldots$ & 3.0 & 1665 & 95.57 & 41.53 & 0.40 & 76.30 & 41.59 & 0.45 & 0.024 & 0.1 & $\mathrm{~B}$ \\
\hline \multirow[t]{3}{*}{ G045.071+0.134 } & $\cdots$ & 4.7 & 1665 & 13.69 & 53.94 & 0.39 & 9.17 & 54.21 & 0.28 & 0.008 & $0.5 \rightarrow 5.0$ & $\mathrm{C}$ \\
\hline & & & 1667 & 6.18 & 53.53 & 0.28 & 2.16 & 55.26 & 0.30 & 0.033 & $2.9 \rightarrow 4.9$ & $\mathrm{C}$ \\
\hline & & & & 5.60 & 55.32 & 0.34 & 3.54 & 56.33 & 0.55 & 0.008 & $1.6 \rightarrow 5.2$ & $\mathrm{C}$ \\
\hline G045.122+0.133 & $\ldots$ & 6.0 & 1667 & 1.90 & 52.98 & 0.38 & 2.08 & 52.18 & 0.36 & 0.005 & -2.3 & $\mathrm{~B}$ \\
\hline \multirow[t]{2}{*}{ G045.465+0.047 } & $\ldots$ & 5.8 & 1665 & 18.26 & 65.97 & 0.41 & 4.32 & 68.18 & 0.54 & 0.011 & 3.7 & $\mathrm{C}$ \\
\hline & & & 1665 & 14.51 & 65.34 & 0.47 & 1.14 & 67.52 & 0.52 & 0.033 & 3.7 & $\mathrm{C}$ \\
\hline G049.469-0.370 & W51 & 7.3 & 1720 & 1.06 & 64.01 & 0.55 & 0.70 & 64.80 & 0.55 & 0.021 & 3.3 & B \\
\hline \multirow[t]{5}{*}{ G049.488-0.387 } & W51 M/S & 6.5 & 1665 & 70.41 & 59.24 & 0.49 & 164.62 & 58.25 & 0.57 & 0.032 & $-1.1 \rightarrow-1.7$ & $\mathrm{C}$ \\
\hline & & & 1720 & 38.30 & 56.97 & 0.53 & 87.99 & 58.16 & 0.44 & 0.001 & 5.0 & $\mathrm{~B}$ \\
\hline & & & & 25.74 & 58.60 & 0.50 & 61.24 & 59.55 & 0.72 & 0.001 & 4.0 & $\mathrm{~B}$ \\
\hline & & & & 5.87 & 55.76 & 0.46 & 7.94 & 56.47 & 0.40 & 0.013 & 3.0 & $\mathrm{~B}$ \\
\hline & & & & 1.96 & 52.64 & 0.31 & 2.42 & 53.47 & 0.44 & 0.013 & 3.5 & B \\
\hline \multirow[t]{3}{*}{ G069.540-0.976 } & ON 1 & 1.3 & 1665 & 4.50 & 13.97 & 0.50 & 15.48 & 11.86 & 0.27 & 0.127 & -3.6 & $\mathrm{~B}$ \\
\hline & & & & 0.64 & 10.87 & 0.29 & 0.84 & 10.74 & 0.43 & 0.074 & -0.2 & $\mathrm{~B}$ \\
\hline & & & & 10.21 & 13.24 & 0.40 & 22.65 & 13.12 & 0.41 & 0.030 & $-0.2 \rightarrow-3.9$ & $\mathrm{C}$ \\
\hline \multirow[t]{3}{*}{ G070.293+1.601 } & K3-50 & 8.0 & 1665 & 2.37 & -21.28 & 0.93 & 2.57 & -22.78 & 2.30 & 0.006 & -2.5 & $\mathrm{~B}$ \\
\hline & & & & 11.52 & -19.76 & 0.26 & 5.64 & -21.27 & 0.46 & 0.008 & -2.5 & $\mathrm{C}$ \\
\hline & & & 1667 & 3.52 & -20.15 & 0.35 & 3.45 & -21.07 & 0.41 & 0.010 & -2.6 & $\mathrm{C}$ \\
\hline \multirow{2}{*}{ G081.721+0.571 } & W75 S & 2.0 & 1665 & 7.61 & -1.29 & 0.27 & 1.40 & -4.00 & 0.28 & 0.102 & -4.6 & $\mathrm{~B}$ \\
\hline & & & & 21.58 & 1.31 & 0.35 & 3.90 & 5.19 & 0.27 & 0.023 & $2.8 \rightarrow 9.3$ & $\mathrm{C}$ \\
\hline \multirow[t]{5}{*}{ G081.871+0.781 } & W75 N & 2.0 & 1665 & 14.61 & 9.35 & 0.20 & 48.50 & 12.47 & 0.30 & 0.007 & 5.3 & B \\
\hline & & & & 16.92 & 5.16 & 0.38 & 5.32 & 5.23 & 0.36 & 0.022 & 0.1 & $\mathrm{~B}$ \\
\hline & & & 1667 & 26.82 & 9.34 & 0.36 & 18.80 & 9.28 & 0.32 & 0.015 & -0.2 & $\mathrm{~B}$ \\
\hline & & & & 0.72 & 10.05 & 0.39 & 0.70 & 1.06 & 0.55 & 0.037 & -25.4 & $D^{f}$ \\
\hline & & & & 1.84 & 5.44 & 0.34 & 0.97 & 8.10 & 0.12 & 0.104 & $7.5 \rightarrow 11.3$ & $\mathrm{C}$ \\
\hline \multirow[t]{3}{*}{ G109.871+2.114 } & Cep A & 0.7 & 1665 & 0.29 & -5.05 & 0.62 & 0.29 & -7.10 & 0.82 & 0.135 & -3.5 & $\mathrm{~B}$ \\
\hline & & & & 9.31 & -16.22 & 0.28 & 7.28 & -14.23 & 0.28 & 0.006 & 3.4 & $\mathrm{C}$ \\
\hline & & & 1667 & 3.78 & -15.77 & 0.37 & 4.34 & -14.64 & 0.30 & 0.012 & 3.2 & $\mathrm{~B}$ \\
\hline G111.533+0.757 & NGC 7538 & 2.8 & 1665 & 2.78 & -54.33 & 0.33 & 2.38 & -52.99 & 0.33 & 0.005 & 2.3 & $\mathrm{~B}$ \\
\hline \multirow[t]{5}{*}{ G133.946+1.064 } & W3 OH & 2.2 & 1612 & 1.30 & -43.77 & 0.54 & 6.27 & -42.20 & 0.45 & 0.033 & $3.6 \rightarrow 6.7$ & $\mathrm{C}$ \\
\hline & & & 1667 & 25.17 & -44.45 & 0.58 & 21.27 & -42.19 & 0.31 & 0.104 & 6.4 & $\mathrm{~B}$ \\
\hline & & & & 1.92 & -47.76 & 0.36 & 0.60 & -47.82 & 0.26 & 0.026 & -0.2 & $\mathrm{~B}$ \\
\hline & & & 1720 & 0.41 & -44.38 & 0.34 & 5.29 & -43.12 & 0.27 & 0.066 & 5.3 & $\mathrm{~B}$ \\
\hline & & & & 10.39 & -45.56 & 0.49 & 7.55 & -44.72 & 0.66 & 0.024 & $2.2 \rightarrow 12.0$ & $\mathrm{C}$ \\
\hline
\end{tabular}


Table 1-Continued

\begin{tabular}{|c|c|c|c|c|c|c|c|c|c|c|c|c|}
\hline Source & Alias & $\begin{array}{l}\text { Dist. }^{\mathrm{a}} \\
(\mathrm{kpc})\end{array}$ & $\begin{array}{l}\text { Freq. } \\
\text { (MHz) }\end{array}$ & $\begin{array}{r}S^{\mathrm{b}} \\
(\mathrm{Jy})\end{array}$ & $\begin{array}{c}-\mathrm{LCP}- \\
v_{\mathrm{LSR}}^{\mathrm{b}} \\
\left(\mathrm{km} \mathrm{s}^{-1}\right)\end{array}$ & $\begin{array}{c}\Delta v^{\mathrm{b}} \\
\left(\mathrm{km} \mathrm{s}^{-1}\right)\end{array}$ & $\begin{array}{r}S^{\mathrm{b}} \\
(\mathrm{Jy})\end{array}$ & $\begin{array}{c}\mathrm{RCP} \\
v_{\mathrm{LSR}}{ }^{\mathrm{b}} \\
\left(\mathrm{km} \mathrm{s}^{-1}\right)\end{array}$ & $\begin{array}{c}\Delta v^{\mathrm{b}} \\
\left(\mathrm{km} \mathrm{s}^{-1}\right)\end{array}$ & $\begin{array}{c}\Delta \theta^{\mathrm{c}} \\
(\operatorname{arcsec})\end{array}$ & $\begin{array}{c}B^{\mathrm{d}} \\
(\mathrm{mG})\end{array}$ & Grade $^{\mathrm{e}}$ \\
\hline G213.706-12.60 & Mon R2 & 0.9 & 1665 & 0.12 & 12.36 & 0.46 & 0.62 & 10.37 & 0.38 & 0.067 & -3.4 & $\mathrm{~B}$ \\
\hline G341.219-0.212 & $\ldots$ & 3.2 & 1665 & 3.49 & -40.85 & 0.45 & 10.06 & -37.40 & 0.33 & 0.027 & 5.8 & $\mathrm{~B}$ \\
\hline G343.128-0.063 & $\cdots$ & 3.1 & 1665 & 86.65 & -31.74 & 0.38 & 13.64 & -30.69 & 0.33 & 0.024 & $1.8 \rightarrow 5.5$ & $\mathrm{C}$ \\
\hline \multirow[t]{3}{*}{ G344.581-0.022 } & $\ldots$ & 0.6 & 1665 & 0.82 & 0.90 & 0.60 & 0.57 & -0.78 & 0.80 & 0.046 & -2.8 & B \\
\hline & & & & 2.23 & -4.40 & 0.69 & 18.57 & -2.33 & 0.58 & 0.060 & $0.7 \rightarrow 3.9$ & C \\
\hline & & & & 14.33 & -2.62 & 0.68 & 0.85 & 1.34 & 0.34 & 0.110 & $4.2 \rightarrow 6.7$ & $\mathrm{C}$ \\
\hline G345.003-0.224 & $\ldots$ & 2.9 & 1720 & 52.64 & -29.24 & 0.33 & 2.16 & -28.80 & 0.44 & 0.008 & 1.9 & $\mathrm{~B}$ \\
\hline \multirow[t]{2}{*}{ G345.011+1.792 } & $\ldots$ & 2.2 & 1665 & 0.68 & -18.99 & 0.27 & 20.22 & -20.44 & 0.41 & 0.083 & -2.5 & $\mathrm{~B}$ \\
\hline & & & & 30.84 & -22.74 & 0.37 & 10.32 & -19.72 & 0.25 & 0.105 & $3.3 \rightarrow 5.1$ & $\mathrm{C}$ \\
\hline \multirow[t]{3}{*}{ G345.505+0.347 } & $\ldots$ & 2.1 & 1665 & 10.91 & -17.32 & 0.35 & 4.37 & -18.01 & 0.27 & 0.106 & $-1.2 \rightarrow-1.8$ & $\mathrm{C}$ \\
\hline & & & 1667 & 2.37 & -12.74 & 0.35 & 4.98 & -12.69 & 0.32 & 0.013 & 0.1 & $\mathrm{~B}$ \\
\hline & & & & 1.15 & -21.58 & 0.37 & 3.78 & -21.25 & 0.31 & 0.011 & 0.9 & $\mathrm{~B}$ \\
\hline G345.699-0.090 & $\ldots$ & 1.6 & 1665 & 0.52 & -8.47 & 0.29 & 3.58 & -8.22 & 0.22 & 0.091 & 0.4 & $\mathrm{~B}$ \\
\hline G347.628+0.149 & $\ldots$ & 9.8 & 1612 & 3.02 & -97.16 & 0.27 & 5.89 & -96.42 & 0.27 & 0.014 & 3.1 & $\mathrm{~B}$ \\
\hline \multirow[t]{2}{*}{ G348.549-0.978 } & $\cdots$ & 2.2 & 1665 & 2.72 & -19.87 & 0.46 & 6.52 & -19.84 & 0.44 & 0.040 & $0.1 \rightarrow 11.5$ & $\mathrm{C}$ \\
\hline & & & 1720 & 4.74 & -12.77 & 0.36 & 4.49 & -13.38 & 0.38 & 0.036 & -2.6 & $\mathrm{~B}$ \\
\hline G350.011-1.341 & $\ldots$ & 3.1 & 1665 & 5.33 & -19.74 & 0.29 & 3.56 & -19.32 & 0.24 & 0.023 & 0.7 & B \\
\hline \multirow[t]{2}{*}{ G351.161+0.697 } & NGC 6334 B & 2.3 & 1667 & 20.11 & -9.72 & 0.32 & 78.79 & -9.64 & 0.22 & 0.028 & 0.2 & $\mathrm{~B}$ \\
\hline & & & & 7.37 & -15.29 & 0.29 & 5.08 & -15.24 & 0.30 & 0.006 & 0.1 & $\mathrm{~B}$ \\
\hline \multirow[t]{3}{*}{ G351.416+0.646 } & NGC $6334 \mathrm{~F}$ & 2.0 & 1665 & 184.98 & -8.87 & 0.32 & 31.40 & -11.99 & 0.57 & 0.045 & -5.3 & $\mathrm{~B}$ \\
\hline & & & 1667 & 47.77 & -9.26 & 0.27 & 54.05 & -11.11 & 0.28 & 0.022 & $-2.2 \rightarrow-5.2$ & $\mathrm{C}$ \\
\hline & & & 1720 & 84.48 & -9.84 & 0.32 & 61.20 & -10.58 & 0.34 & 0.020 & -3.1 & $\mathrm{~B}$ \\
\hline G351.582-0.352 & $\ldots$ & 6.7 & 1665 & 2.73 & -90.97 & 0.32 & 5.82 & -93.87 & 0.25 & 0.019 & -4.9 & $\mathrm{~B}$ \\
\hline \multirow[t]{8}{*}{ G351.775-0.538 } & $\ldots$ & 2.7 & 1665 & 2.94 & -25.62 & 0.46 & 4.80 & -27.86 & 0.39 & 0.025 & -3.8 & $\mathrm{~A}$ \\
\hline & & & & 777.34 & -1.95 & 0.40 & 106.28 & -1.85 & 0.47 & 0.013 & 0.2 & $\mathrm{~B}$ \\
\hline & & & & 4.09 & -8.04 & 0.29 & 6.92 & -7.84 & 1.06 & 0.063 & 0.3 & $\mathrm{~B}$ \\
\hline & & & & 54.35 & -6.88 & 0.40 & 4.42 & -10.16 & 0.57 & 0.047 & $-5.6 \rightarrow-6.5$ & $\mathrm{C}$ \\
\hline & & & 1667 & 3.06 & -26.15 & 0.43 & 4.23 & -27.44 & 0.44 & 0.014 & -3.7 & $\mathrm{~A}$ \\
\hline & & & & 58.40 & -6.97 & 0.30 & 1.85 & -4.95 & 0.38 & 0.029 & 5.7 & $\mathrm{~B}$ \\
\hline & & & & 10.02 & -5.55 & 0.27 & 2.62 & -5.83 & 0.29 & 0.024 & -0.8 & $\mathrm{~B}$ \\
\hline & & & & 1.51 & 0.37 & 0.42 & 1.49 & -0.16 & 0.67 & 0.037 & $-0.3 \rightarrow-1.5$ & $\mathrm{C}$ \\
\hline G353.410-0.361 & $\ldots$ & 3.8 & 1665 & 19.83 & -19.56 & 0.29 & 3.30 & -19.50 & 0.48 & 0.033 & $0.1 \rightarrow 2.4$ & $\mathrm{C}$ \\
\hline G355.345+0.146 & $\ldots$ & 23.1 & 1665 & 17.07 & 19.71 & 0.42 & 15.34 & 16.54 & 0.86 & 0.006 & $-3.4 \rightarrow-5.4$ & $\mathrm{C}$ \\
\hline \multirow[t]{2}{*}{ G359.138+0.032 } & $\ldots$ & 3.1 & 1665 & 4.60 & -0.21 & 0.40 & 3.32 & -2.98 & 0.71 & 0.014 & -4.7 & $\mathrm{~B}$ \\
\hline & & & & 2.92 & -6.20 & 0.37 & 1.39 & -6.09 & 0.31 & 0.047 & 0.2 & B \\
\hline
\end{tabular}




\section{Zeeman Pairs}

\subsection{Distances}

The distances given in Tables 1 and 2 are kinematic distances assuming a flat rotation curve with $R_{0}=8.5 \mathrm{kpc}$ and $\Theta(R)=220 \mathrm{~km} \mathrm{~s}^{-1}$, except as noted in the text. For sources inside the solar circle in the first and fourth Galactic quadrants, there is a distance ambiguity. For a number of these sources, the kinematic distance ambiguity is resolved from $\mathrm{H} \mathrm{I}$ absorption observations by Fish et al. (2003). That paper also provides an analysis of the scale height of known UCH II regions (embedded in massive SFRs) above the Galactic plane, including an analysis of the accuracy of resolving the distance ambiguity to a UCH II region given only the kinematic distances and Galactic latitude of the region. When the distance was not known by any other method, the Galactic latitude was used to resolve the kinematic distance ambiguity toward massive SFRs only when the difference between the near and far kinematic distances exceeds $4.5 \mathrm{kpc}$, corresponding to $90 \%$ predictive accuracy (Fish et al. 2003). The majority of these sources are located in the fourth Galactic quadrant.

When evaluating kinematic distances, we assumed that massive SFRs have a peculiar motion of $\lesssim 10 \mathrm{~km} \mathrm{~s}^{-1}$. This freedom is important to placing a few sources (e.g., G355.345+0.146) at a sensible distance. Nevertheless, it is possible for peculiar motions to exceed $10 \mathrm{~km} \mathrm{~s}^{-1}$. Hydroxyl and $\mathrm{H}_{2} \mathrm{CO}$ absorption measurements toward G10.624-0.385 and other sources in the W31 complex demonstrate deviations from the velocities expected from the rotation model of as much as 36 $\mathrm{km} \mathrm{s}^{-1}$ (Wilson 1974).

For sources whose median LSR velocity of $\mathrm{OH}$ maser emission is close to $0 \mathrm{~km} \mathrm{~s}^{-1}$, the near kinematic distance is often unrealistically close. Since the angular window radius as described in $\S 3$ is inversely proportional to the assumed distance to the source, a source assumed to be much nearer than it is would be partitioned into windows encompassing too large of an angular area. The resulting windows would be few in number and contain a large number of maser lines. The effect is amplified for especially near distances (a few hundred parsecs or less), where a small velocity error translates into a large fractional distance error and therefore a large fractional error in the window size. To be consistent (if somewhat arbitrary) in handling these extremely near sources ( $\ll 2 \mathrm{kpc}$ ), we used the distance produced by averaging the kinematic distance derived from the median $\mathrm{OH}$ maser velocity and $2.0 \mathrm{kpc}$. Since massive SFRs are few and far between, it is unlikely

that a massive SFR whose distance is not independently known is actually located within $1 \mathrm{kpc}$ of the Sun.

\subsection{Satellite-Line Field Splitting}

Unlike the main-line (1665 and $1667 \mathrm{MHz}$ ) transitions, which split into only one pair of $\sigma$ components, the satellite-line transtitions split into three pairs with line intensities in the ratio 6:3:1 (Davies 1974). As mentioned in a footnote to Table 1, the Zeeman splitting coefficient 
Table 1 - Continued

\begin{tabular}{|c|c|c|c|c|c|c|c|c|c|c|c|c|}
\hline Source & Alias & $\begin{array}{l}\text { Dist. }^{a} \\
(\mathrm{kpc})\end{array}$ & $\begin{array}{l}\text { Freq. } \\
(\mathrm{MHz})\end{array}$ & $\begin{array}{r}S^{\mathrm{b}} \\
(\mathrm{Jy})\end{array}$ & $\begin{array}{c}\mathrm{LCP}- \\
v_{\mathrm{LSR}}{ }^{\mathrm{b}} \\
\left(\mathrm{km} \mathrm{s}^{-1}\right)\end{array}$ & $\begin{array}{c}\Delta v^{\mathrm{b}} \\
\left(\mathrm{km} \mathrm{s}^{-1}\right)\end{array}$ & $\begin{array}{r} \\
S^{\mathrm{b}} \\
(\mathrm{Jy})\end{array}$ & $\begin{array}{c}-\mathrm{RCP} \\
v_{\mathrm{LSR}}{ }^{\mathrm{b}} \\
\left(\mathrm{km} \mathrm{s}^{-1}\right)\end{array}$ & $\begin{array}{c}\Delta v^{\mathrm{b}} \\
\left(\mathrm{km} \mathrm{s}^{-1}\right)\end{array}$ & $\begin{array}{c}\Delta \theta^{\mathrm{c}} \\
(\operatorname{arcsec})\end{array}$ & $\begin{array}{c}B^{\mathrm{d}} \\
(\mathrm{mG})\end{array}$ & Grade $^{\mathrm{e}}$ \\
\hline G359.436-0.103 & $\ldots$ & 8.2 & 1665 & 4.66 & -51.83 & 0.48 & 2.60 & -52.11 & 0.63 & 0.032 & -0.5 & B \\
\hline
\end{tabular}

${ }^{\mathrm{a}}$ Kinematic distances assuming $R_{0}=8.5 \mathrm{kpc}$ and $\Theta_{0}=220 \mathrm{~km} \mathrm{~s}^{-1}$, unless note in text $(\S 4.3)$ says otherwise.

${ }^{\mathrm{b}}$ Peak flux density, center velocity, and line width (FWHM) of left- and right-circular polarized lines, respectively, as determined by Gaussian fit.

${ }^{\mathrm{c}}$ Angular offset between LCP and RCP lines. When a range of values is given for the magnetic field $(B)$, the offset is stated for the lines of peak flux density in each polarization.

${ }^{\mathrm{d}}$ Full magnitude of $B$ field. The sign indicates the direction of the line-of-sight component (positive pointing away from Sun). The ratio of the velocity separation between RCP and LCP components to $B$ field was taken to be $0.590,0.354,0.236$, and $0.236 \mathrm{~km} \mathrm{~s}^{-1} \mathrm{mG}^{-1}$ for the 1665 , 1667,1612 , and $1720 \mathrm{MHz}$ transitions, respectively. See $\S 4.2$ for further details. When a range is given, the extrema represent the minimum and maximum field strength implied by all possible pairings of RCP and LCP lines.

${ }^{\mathrm{e}}$ See text (§3) for description.

${ }^{\mathrm{f}}$ Likely spurious. See $\S 4.3$ for details. 
for the satellite-line $(1612$ and $1720 \mathrm{MHz})$ transitions was assumed to be $0.236 \mathrm{~km} \mathrm{~s}^{-1} \mathrm{mG}^{-1}$. This splitting coefficient is correct for the intensity-weighted average of the three lines in each polarization. However, maser amplification may be greatest for the strongest line in each triad. If only the strongest line is seen after amplification, the splitting coefficient would be $0.114 \mathrm{~km} \mathrm{~s}^{-1}$

$\mathrm{mG}^{-1}$, or approximately half of the intensity-weighted value. Indeed, the splitting coefficient could be between the two extremal values, as would be consistent with amplification of all three $\sigma$ components in a partially-saturated maser. A comparison of the magnetic field strengths inferred from $1720 \mathrm{MHz}$ pairs in W3(OH) listed in Table 1 with those shown on VLBI maps (Bloemhof, Reid, \& Moran 1992) is more consistent with a splitting coefficient closer to $0.236 \mathrm{~km} \mathrm{~s}^{-1} \mathrm{mG}^{-1}$. Analysis of field strengths inferred from 1612 and $1720 \mathrm{MHz}$ Zeeman pairs in all sources also supports a large splitting coefficient (see §4.4). In any case, the direction of the line-of-sight component of the field is unaffected.

\subsection{Notes on Specific Sources}

G000.670-0.058: The separation between the LCP and RCP components of the pair exceeds the assumed $3 \times 10^{15} \mathrm{~cm}$ correlation length by less than a factor of two.

G005.886-0.393: The two pairs receiving an A grade are separated by approximately 0 " .3 .

G009.622+0.195: We adopt a distance of $5.7 \mathrm{kpc}$ to this source, consistent with observations by Scoville et al. (1987) and Hofner et al. (1994), although the distance obtained kinematically could be as near as $0.7 \mathrm{kpc}$. The second Zeeman pair listed in Table 1, offset by about $5^{\prime \prime}$ from the other two pairs, is in a separate maser site within the same massive star-forming cluster. Additional lines for the first pair in table 1 receiving a grade of $\mathrm{C}$ consist of an LCP line of $1.65 \mathrm{Jy}$ at -3.97 $\mathrm{km} \mathrm{s}^{-1}$ and an RCP line at $7.85 \mathrm{Jy}$ and $1.22 \mathrm{~km} \mathrm{~s}^{-1}$. For the second such pair, there are four additional LCP lines: $0.79 \mathrm{Jy}$ at $7.04 \mathrm{~km} \mathrm{~s}^{-1}, 0.39 \mathrm{Jy}$ at $6.50 \mathrm{~km} \mathrm{~s}^{-1}, 0.47 \mathrm{Jy}$ at $6.09 \mathrm{~km} \mathrm{~s}^{-1}$, and $1.32 \mathrm{Jy}$ at $5.49 \mathrm{~km} \mathrm{~s}^{-1}$.

G012.680-0.181: Additional lines for the 1665 pair receiving a grade of $\mathrm{C}$ consist of two RCP lines: $2.23 \mathrm{Jy}$ at $60.71 \mathrm{~km} \mathrm{~s}^{-1}$ and $2.58 \mathrm{Jy}$ at $61.17 \mathrm{~km} \mathrm{~s}^{-1}$. For the 1667 pair, there are two additional LCP lines of $2.92 \mathrm{Jy}$ at $64.81 \mathrm{~km} \mathrm{~s}^{-1}$ and $2.28 \mathrm{Jy}$ at $62.76 \mathrm{~km} \mathrm{~s}^{-1}$ and one additional RCP line of $3.30 \mathrm{Jy}$ at $62.15 \mathrm{~km} \mathrm{~s}^{-1}$. From observations using the $43 \mathrm{~m}$ telescope at Green Bank, Zheng (1991) reports two Zeeman pairs of around $-5 \mathrm{mG}$ each. The $4 \mathrm{kpc}$ distance assumed for W33 B and W33 A is the kinematic distance taken from Haschick \& Ho (1983).

G020.081-0.135: There is an additional LCP line of $4.44 \mathrm{Jy}$ at $45.47 \mathrm{~km} \mathrm{~s}^{-1}$ for the pair receiving a $\mathrm{C}$ grade.

G032.744-0.076: There is an additional RCP line of $0.24 \mathrm{Jy}$ at $40.24 \mathrm{~km} \mathrm{~s}^{-1}$ for the pair receiving a $\mathrm{C}$ grade.

G034.257+0.154: There is an additional LCP line of $2.24 \mathrm{Jy}$ at $57.97 \mathrm{~km} \mathrm{~s}^{-1}$ for the pair 
receiving a $\mathrm{C}$ grade. This source is one of three in the G34.3+0.2 complex. The magnetic field structure of this cometary H II region, source C in Zheng, Reid, \& Moran (2000), is unclear. The magnetic field of source B (G034.258+0.153) is ordered and pointing primarily away from the Sun.

G035.024+0.350: There is an additional LCP line of $0.54 \mathrm{Jy}$ at $43.83 \mathrm{~km} \mathrm{~s}^{-1}$ and an RCP line at $1.83 \mathrm{Jy}$ and $44.63 \mathrm{~km} \mathrm{~s}^{-1}$.

G035.197-0.743: There is an additional LCP line of $1.04 \mathrm{Jy}$ at $26.35 \mathrm{~km} \mathrm{~s}^{-1}$.

G035.577-0.029: In each of the two pairs, the angular offset between the LCP and RCP lines is consistent with a separation of about $2 \times 10^{16} \mathrm{~cm}$, assuming the kinematic distance. There are two additional LCP lines of $2.85 \mathrm{Jy}$ at $51.89 \mathrm{~km} \mathrm{~s}^{-1}$ and $2.61 \mathrm{Jy}$ at $49.91 \mathrm{~km} \mathrm{~s}^{-1}$ for the second pair receiving a $\mathrm{D}$ grade.

G040.622-0.137: There is an additional RCP line of $1.04 \mathrm{Jy}$ at $34.76 \mathrm{~km} \mathrm{~s}^{-1}$ for the 1665 pair receiving a grade of $\mathrm{C}$. For the 1667 pair, there are two additional LCP lines: $2.82 \mathrm{Jy}$ at 31.53 $\mathrm{km} \mathrm{s}^{-1}$ and $1.34 \mathrm{Jy}$ at $30.85 \mathrm{~km} \mathrm{~s}^{-1}$.

G045.071+0.134: For the 1665 pair, there are two additional LCP lines of 6.24 Jy at 53.57 $\mathrm{km} \mathrm{s}^{-1}$ and $8.53 \mathrm{Jy}$ at $52.95 \mathrm{~km} \mathrm{~s}^{-1}$ and one additional RCP line of $7.21 \mathrm{Jy}$ at $55.91 \mathrm{~km} \mathrm{~s}^{-1}$. For the first 1667 pair, there is an additional RCP line of $7.21 \mathrm{Jy}$ at $55.91 \mathrm{~km} \mathrm{~s}^{-1}$. For the second 1667 pair, there is an additional LCP line of $2.97 \mathrm{Jy}$ at $55.78 \mathrm{~km} \mathrm{~s}^{-1}$ and an RCP line of $0.91 \mathrm{Jy}$ at $57.17 \mathrm{~km} \mathrm{~s}^{-1}$.

G045.465+0.047: The LCP and RCP lines of each of the two pairs are located in a circle of $3 \times 10^{15} \mathrm{~cm}$. While the pairing of these lines is theoretically ambiguous, we feel that the identical magnetic fields implied by the listed pairings and the small velocity difference between these pairings ( $0.65 \mathrm{~km} \mathrm{~s}^{-1}$, less than the $0.8 \mathrm{~km} \mathrm{~s}^{-1}$ turbulent velocity differences expected within a masing cloud) are sufficient to justify our claimed magnetic field values.

G049.469-0.370: In 18-cm VLA observations, Gaume \& Mutel (1987) also find a Zeeman pair with a positive magnetic field. For more information, see the notes for the next source.

G049.488-0.387: Gaume \& Mutel (1987) find about half a dozen Zeeman pairs in the W51 complex around regions e1 and e2, all indicating a positive magnetic field. In 5-cm VLBI observations, Desmurs \& Baudry (1998) find a Zeeman pair between regions e1 and e2. However, using the VLBA Argon, Reid, \& Menten (2002) find two very strong $1665 \mathrm{MHz}$ Zeeman pairs indicating magnetic fields of -21 and $-19.8 \mathrm{mG}$. The kinematic distance is taken from Caswell (2001). For the 1665 pair, there is an additional LCP line of $59.42 \mathrm{Jy}$ at $58.90 \mathrm{~km} \mathrm{~s}^{-1}$.

G069.540-0.976: For the 1665 pair receiving a $\mathrm{C}$ grade, there are two additional LCP lines: $5.07 \mathrm{Jy}$ at $15.45 \mathrm{~km} \mathrm{~s}^{-1}$ and $3.17 \mathrm{Jy}$ at $15.06 \mathrm{~km} \mathrm{~s}^{-1}$. Preliminary (as yet unpublished) VLBA 18-cm observations by the authors of this paper and VLBI 5-cm maps of Desmurs \& Baudry (1998) confirm that the magnetic field is consistently oriented toward the Sun across the source.

G070.293+1.601: For the 1665 pair receiving a $\mathrm{C}$ grade, there is an additional LCP line of 
$4.03 \mathrm{Jy}$ at $-20.39 \mathrm{~km} \mathrm{~s}^{-1}$. For the 1667 pair, there is an additional LCP line of $2.46 \mathrm{Jy}$ at -20.80 $\mathrm{km} \mathrm{s}^{-1}$. However, the two grade-C pairs in the table meet the self-consistency criteria $\left(\Delta \theta<0^{\prime \prime} .2\right.$, $\Delta v_{c}<0.2 \mathrm{~km} \mathrm{~s}^{-1}, \Delta B<0.4 \mathrm{mG}$ between pairs in the two transitions).

G081.721+0.571: Both this source and G081.871+0.781, believed to be in the same complex, have average LSR velocities of a few $\mathrm{km} \mathrm{s}^{-1}$ positive. This puts these sources near the tangent point at this Galactic longitude with a large uncertainty in distance. We adopt the Dickel, Dickel, \& Wilson (1978) distance of $2.0 \mathrm{kpc}$ as the distance to the W75 complex. For the pair receiving a grade of C, there are additional LCP lines of $15.48 \mathrm{Jy}$ at $0.93 \mathrm{~km} \mathrm{~s}^{-1}, 8.45 \mathrm{Jy}$ at $0.58 \mathrm{~km} \mathrm{~s}^{-1}$, and $2.44 \mathrm{Jy}$ at $-0.27 \mathrm{~km} \mathrm{~s}^{-1}$ as well as RCP lines of $0.40 \mathrm{Jy}$ at $4.79 \mathrm{~km} \mathrm{~s}^{-1}, 0.65 \mathrm{Jy}$ at $3.53 \mathrm{~km} \mathrm{~s}^{-1}$, and $1.49 \mathrm{Jy}$ at $2.97 \mathrm{~km} \mathrm{~s}^{-1}$.

G081.871+0.781: Preliminary (as yet unpublished) VLBA observations indicate a reversal of the line-of-sight magnetic field direction across the source. The $-25.4 \mathrm{mG}$ pair is not seen with the VLBA. However, a cluster that contains a large number of maser spots with a large velocity spread $\left(\approx 20 \mathrm{~km} \mathrm{~s}^{-1}\right)$ is detected. It is probable that this "pair" is actually two nearby lines whose velocity difference is due to a velocity gradient rather than an extremely large magnetic field. For distance information, see the note for G081.721+0.571.

G109.871+2.114: For the 1665 pair receiving a $\mathrm{C}$ grade, there is an additional LCP line of $13.65 \mathrm{Jy}$ at $-13.90 \mathrm{~km} \mathrm{~s}^{-1}$. However, the ambiguity is resolved by examining the 1667 transition, which is within $0^{\prime \prime} .2$ of the 1665 pair in question. Cohen, Brebner, \& Potter (1990) find a Zeeman pair that implies a magnetic field direction oriented away from the Sun. Monitoring of this Zeeman pair over time shows that the strength of the magnetic field is decreasing.

G111.533+0.757: The distance of $2.8 \mathrm{kpc}$ is taken from Campbell \& Thompson (1984).

G133.946+1.064: For the 1612 pair, there is an addition RCP line of $3.76 \mathrm{Jy}$ at $-42.92 \mathrm{~km} \mathrm{~s}^{-1}$. For the 1720 line receiving a $\mathrm{C}$ grade, there is an additional LCP line of $6.88 \mathrm{Jy}$ at $-45.23 \mathrm{~km} \mathrm{~s}^{-1}$ and two additional RCP lines: $4.47 \mathrm{Jy}$ at $-42.72 \mathrm{~km} \mathrm{~s}^{-1}$ and $7.47 \mathrm{Jy}$ at $-44.36 \mathrm{~km} \mathrm{~s}^{-1}$. Previous VLBI observations have clearly established that the magnetic field is predominantly oriented away from the Sun (García-Barreto et al. 1988). The $2.2 \mathrm{kpc}$ distance used for W3(OH) is based on OB star luminosities from Humphreys (1978).

G343.128-0.063: There is an additional LCP line of $54.52 \mathrm{Jy}$ at $-33.93 \mathrm{~km} \mathrm{~s}^{-1}$.

G344.581-0.022: The near kinematic distance of $0.6 \mathrm{kpc}$ (Forster \& Caswell 1989) is too close, as evidenced by the cluttered spectra produced in circles of radius $3 \times 10^{15} \mathrm{~cm}$, but no pairs are seen at all at the far kinematic distance of $15.8 \mathrm{kpc}$. A distance of $2.0 \mathrm{kpc}$ was used, which produces circles of radius $10^{15} \mathrm{~cm}$ assuming the near kinematic distance is correct. For the first pair receiving a $\mathrm{C}$ grade, there is an additional LCP line of $1.99 \mathrm{Jy}$ at $-4.66 \mathrm{~km} \mathrm{~s}^{-1}$ and an RCP line of $2.93 \mathrm{Jy}$ at $-4.01 \mathrm{~km} \mathrm{~s}^{-1}$. For the second such pair, there is an additional LCP line of 3.74 Jy at $-1.16 \mathrm{~km} \mathrm{~s}^{-1}$.

G345.011+1.792: For the pair receiving a C grade, there is an additional LCP line of 3.39 Jy 
at $-21.67 \mathrm{~km} \mathrm{~s}^{-1}$.

G345.505+0.347: For the pair receiving a $\mathrm{C}$ grade, there is an additional LCP line of 2.72 Jy at $-16.92 \mathrm{~km} \mathrm{~s}^{-1}$.

G348.549-0.978: For the pair receiving a $\mathrm{C}$ grade, there are two additional RCP lines: 4.46 Jy at $-13.11 \mathrm{~km} \mathrm{~s}^{-1}$ and $2.64 \mathrm{Jy}$ at $-19.26 \mathrm{~km} \mathrm{~s}^{-1}$.

G351.416+0.646: For the pair receiving a C grade, there is an additional LCP line of 22.29 Jy at $-10.33 \mathrm{~km} \mathrm{~s}^{-1}$.

G351.775-0.538: For the 1665 pair receiving a $\mathrm{C}$ grade, there is an additional RCP line of $3.04 \mathrm{Jy}$ at $-10.72 \mathrm{~km} \mathrm{~s}^{-1}$. For the 1667 pair receiving a $\mathrm{C}$ grade, there is an additional RCP line of $1.11 \mathrm{Jy}$ at $0.25 \mathrm{~km} \mathrm{~s}^{-1}$. Preliminary VLBA observations indicate a reversal of the line-of-sight magnetic field direction across the source (Argon, Reid, \& Menten 2002).

G353.410-0.361: There is an additional LCP line of $3.55 \mathrm{Jy}$ at $-20.56 \mathrm{~km} \mathrm{~s}^{-1}$ and an RCP line of $2.34 \mathrm{Jy}$ at $-19.12 \mathrm{~km} \mathrm{~s}^{-1}$. Caswell (2001) finds evidence for a magnetic field pointing in the opposite direction based on 6030- and 6035- $\mathrm{MHz} \mathrm{OH}$ masers.

G355.345+0.146: The rotation curve is quite flat in this part of the galaxy. The distance of $23.1 \mathrm{kpc}$ was derived assuming a velocity of $+7.5 \mathrm{~km} \mathrm{~s}^{-1}$, or $10 \mathrm{~km} \mathrm{~s}^{-1}$ less than the actual average value as deduced from $\mathrm{OH}$ maser emission. (Recall that a positive velocity in the fourth Galactic quadrant indicates that the source is located outside the solar circle, or at least $17 \mathrm{kpc}$ away at this Galactic longitude.) The distance deduced from the actual average velocity of $+17.5 \mathrm{~km} \mathrm{~s}^{-1}$ is unphysically large, assuming a flat rotation curve with $R_{0}=8.5 \mathrm{kpc}$ and $\Theta(R)=220 \mathrm{~km} \mathrm{~s}^{-1}$. There are two additional LCP lines: $15.38 \mathrm{Jy}$ at $18.95 \mathrm{~km} \mathrm{~s}^{-1}$ and $15.84 \mathrm{Jy}$ at $18.55 \mathrm{~km} \mathrm{~s}^{-1}$.

\subsection{Distribution of Magnetic Field Strengths}

A histogram of the inferred magnetic field strengths for 75 Zeeman pairs from Table 1 is included in Figure 2. Only those Zeeman pairs implying a single value of the magnetic field strength are included, and the $-25.4 \mathrm{mG}$ pair in G081.871+0.781 is omitted (see $\S 4.3$ ). A Gaussian fit is superimposed on the histogram. The distribution of field strengths has a HWHM of $3.9 \pm 0.1 \mathrm{mG}$ and is centered at $0 \mathrm{mG}$ to within the formal error. This is consistent with average field magnitudes of several milligauss deduced from $\mathrm{OH}$ Zeeman splitting in other studies (e.g., García-Barreto et al. 1988). Note that this field strength is what would be expected for magnetic field strength enhancement of $|B| \propto n^{1 / 2}$ (Mouschovias 1976) from interstellar values of $B$ and $n(\sim 1 \mu \mathrm{G}$, $\sim 1 \mathrm{~cm}^{-3}$ ) to $n \sim 10^{6} \mathrm{~cm}^{-3}$, as is typical for sites of $\mathrm{OH}$ masing (Reid \& Moran 1981).

Of the 15 Zeeman pairs detected at 1612 and $1720 \mathrm{MHz}, 10$ imply a magnetic field strength less than or equal to the HWHM assuming a splitting coefficient of $0.236 \mathrm{~km} \mathrm{~s}^{-1} \mathrm{mG}^{-1}, 3$ imply a field strength greater than the HWHM, and the 2 pairs with a grade of $\mathrm{C}$ in Table 1 are inconclusive 
owing to pairing ambiguities. If a splitting coefficient of $0.114 \mathrm{~km} \mathrm{~s}^{-1} \mathrm{mG}^{-1}$ is assumed, 3 Zeeman pairs imply a magnetic field strength less than the HWHM and 12 imply a greater field strength. Thus, magnetic field strengths deduced from 1612 and $1720 \mathrm{MHz}$ Zeeman pairs are more consistent with values obtained from the main-line transitions when a splitting coefficient value closer to the high value $\left(0.236 \mathrm{~km} \mathrm{~s}^{-1} \mathrm{mG}^{-1}\right)$ is assumed. While we cannot rule out the possibility that the physical requirements for masing at 1612 and $1720 \mathrm{MHz}$ cause satellite-line maser spots to be observed preferentially at locations of increased magnetic field strength, we feel justified in using the larger splitting coefficient.

\subsection{Other $\mathrm{OH}$ Zeeman Studies}

This study was undertaken primarily to investigate Davies's hypothesis that the magnetic fields deduced from $\mathrm{OH}$ Zeeman splitting in massive SFRs exhibit Galactic-scale organization. Preliminary analysis of the data from the Paper I survey yielded 40 massive SFRs for which $\mathrm{OH}$ Zeeman splitting suggested a local field direction (Fish et al. 2002). While the data set was homogeneous, it excluded southern sources, which are not observable from the VLA. To address this, the current study includes $\mathrm{OH}$ Zeeman pairs from a number of other studies as well. Chief among these are the $6 \mathrm{GHz}$ surveys of Baudry et al. (1997) and Caswell \& Vaile (1995), the 1.6 GHz study of Gaume \& Mutel (1987), and the original literature search of Reid \& Silverstein (1990). Two sources for which we have unpublished maps in the 1665 and $1667 \mathrm{MHz} \mathrm{OH}$ transitions with the VLBA have also been included (ON2 N and S269) (in preparation). Other supplementary papers relevant to only one or a small number of our sources are mentioned with the appropriate source(s) in $\S 4.3$. The objective is to compile a list of massive SFRs restricted to those with a predominant magnetic field direction.

When assigning an overall magnetic field direction to a massive SFR, we require that at least two-thirds of all Zeeman pairs for any one source imply the same line-of-sight field direction. In addition to problems associated with a possible magnetic field reversal across the source, the VLA beam is large enough to encompass several maser clumps. While this could potentially blend maser spots, resulting in misidentification of Zeeman pairs, we have striven to obtain a sound collection of Zeeman pairs by discarding all pairs that do not meet the objective criteria outlined in $\S 3$, at the expense of completeness. The even lower spatial resolution of the data taken from $\lambda=5 \mathrm{~cm}$ studies is not a concern since the intensity of RCP and LCP lines in a $6035 \mathrm{MHz}$ pair is typically much more equal than is seen in ground-state Zeeman pairs (Caswell \& Vaile 1995). This fact, combined with a much smaller Zeeman splitting coefficient, makes unambiguous pairing of maser spots easier in the $6 \mathrm{GHz}$ transitions. Thus we do not believe that blending of multiple maser spots in the same beamwidth is a significant source of contamination for the magnetic field measurements in this study. The final sample to be used in $\S 5.2$ comprises 45 of the 53 sources in Table 1, plus 29 from other information. 


\section{Discussion}

A number of historical findings motivated us to study whether or not the Galactic magnetic field is sensed by $\mathrm{OH}$ maser Zeeman splitting. The original hypothesis of a link between the Galactic magnetic field and interstellar OH masers was advanced by Davies (1974), who, noting that his compilation of eight $\mathrm{OH}$ maser sources all displayed a magnetic field consistent with the clockwise direction of Galactic rotation, suggested that the $\mathrm{OH}$ masers traced a circular Galactic magnetic field. For the most part, the $\mathrm{OH}$ sources included in his study are located near the solar circle, precluding investigation of reversals of the large-scale magnetic field direction with Galactocentric radius. Reid \& Silverstein (1990) tested the Davies hypothesis by finding 12 new $\mathrm{OH}$ maser sources, of which 10 had a line-of-sight magnetic field aligned with the direction of Galactic rotation. This seemed to be consistent with Davies's suggestion and a concentric-ring model of the Galactic magnetic field as deduced from the pulsar rotation measure study of Rand \& Kulkarni (1989). However, Rand \& Kulkarni also presented evidence for magnetic field reversals between rings, which was not critically tested in the Reid \& Silverstein study.

\subsection{Preservation of the Galactic Magnetic Field Direction}

Should the magnetic field as deduced from the Zeeman splitting of $\mathrm{OH}$ masers around massive SFRs show any Galactic-scale structure? This would require three important conditions to be satisfied. First, there must be a large-scale Galactic magnetic field, which is likely given pulsar (e.g., Manchester 1974) and extragalactic rotation measure observations (e.g., Sofue et al. 1986). Second, the OH clumps must "remember" the Galactic magnetic field after a magnetic compression of about three orders of magnitude from interstellar values. And third, intra-source reversals of the magnetic field in an individual massive SFR must be sufficiently few so as to not destroy a possible observed large-scale Galactic magnetic field by the inclusion of inferred local magnetic field directions that are opposite to the actual prevailing magnetic field direction near the source.

Of these three conditions, the most difficult to justify on a physical basis is the assumption that the magnetic field direction at a site of massive star-formation would retain its initial (Galactic) direction through collapse. Ammonia observations of $\mathrm{W} 3(\mathrm{OH})$ suggest that the density in $\mathrm{OH}$ maser clumps is $n \approx 5 \times 10^{6} \mathrm{~cm}^{-3}$ (Reid, Myers, \& Bieging 1987), which is a factor of $10^{3-4}$ higher than found in giant molecular clouds and a factor of $10^{6}$ greater than typical values in the ISM. It is unclear why the magnetic field in these clumps would retain the orientation of the field in the giant molecular cloud (GMC) during the compression phase, which may also entail many rotations

of the clumps around the central condensation, and why the GMC would retain the orientation of the interstellar field. 


\subsection{Structure of the Galactic Magnetic Field}

Assuming that the second and third of the above conditions are satisfied, we can investigate whether there exists a large-scale Galactic magnetic field and what form it takes. The simplest imaginable Galactic field would be a circular field with no reversals in the portion of the disk where massive SFRs are found. Our 74-source sample discussed in $\$ 4.5$ do not support this model. A plot of the predominant sense of the magnetic field direction (i.e., clockwise or counterclockwise as viewed from the North Galactic Pole) deduced from the $\mathrm{OH}$ masers near massive SFRs in this study is shown in Figure 3. For a circular magnetic field configuration in the Milky Way, the sense of the line-of-sight orientation (directed toward or away from the Sun) flips across the $l=0, l=180^{\circ}$ line. Noting this, 41 have magnetic fields directed in a clockwise sense as viewed from the North Galactic Pole and 33 have fields directed in a counterclockwise sense. This does not support a uniform circular field model without reversals. Indeed, it would not be inconsistent with a completely random distribution of magnetic field orientations.

The rough equality of sources with clockwise and counterclockwise fields does not preclude an organized field structure. For instance, the magnetic field could be aligned along either concentric rings or spiral arms but with reversals between the rings or arms. Alternatively, the magnetic field as traced by interstellar $\mathrm{OH}$ masers could exhibit organized structure in small (kiloparsec-scale) patches, but not on a Galactic scale. Since our data do not imply a uniform sense of the Galactic magnetic field, a two-point spatial correlation function was calculated on the data in order to test the hypothesis that magnetic field directions of massive SFRs exhibit some degree of correlation over kiloparsec scales. We define the magnetic field orientation of two massive SFRs to be correlated if they are both oriented in a clockwise or counterclockwise Galactic sense. We similarly define a pair to be anti-correlated if the pair consists of one source with a clockwise field and one with a counterclockwise field. The correlation function was defined as the number of pairs of massive SFRs whose fields were correlated in the above sense, divided by the total number of pairs of sources. This function was calculated for a range of intersource distances. Since interpretation of the significance of the correlation function is difficult in isolation, it was compared against the same function evaluated for randomly-generated magnetic-field direction data at the locations of the massive SFRs in our sample. In the random sample, the positions of the maser sources were held fixed, but the line-of-sight field orientation of each source was randomly assigned as toward or away from the Sun. An ensemble of $10^{5}$ such random field distributions was produced. The results are shown in Table 3. The percentages of trial runs showing a lesser and greater degree of correlation do not sum to $100 \%$ because some random distributions of magnetic fields produced identical correlation results.

Since the data exhibit roughly equal likelihood of less and more correlation at the 10 or $20 \mathrm{kpc}$ level, our data do not support the original hypothesis of Davies (1974) that there exists a uniform, clockwise Galactic field. On Galactic scales, pulsar rotation measure studies suggest that field reversals exist between spiral arms (e.g., Han et al. 2002; Rand \& Lyne 1994). This would destroy

any large-scale correlation on the Galactic scale, as spiral arms with oppositely-oriented magnetic 
fields would be included at this scale.

On sub-kiloparsec scales there are a number of source complexes in which two nearby massive SFRs have oppositely-oriented line-of-sight magnetic fields. The W49 complex provides a good example of this: G43.148+0.015 (the Zeeman pair is between the sources W49A/ $R_{2}$ and $R_{3}$ in the classification of De Pree, Mehringer, \& Goss 1997) and W49S have fields directed primarily away from the Sun, while the field of W49N is directed toward the Sun. It is possible that this may result from a tangled field geometry associated with multiple cores in the same complex.

Nevertheless, we do note a possible field organization on a $\sim 2$-kpc scale. Indeed, several patches of field coherence at this scale are noticeable in Figure 3. For instance, there is a cluster of sources with clockwise magnetic fields (i.e., oriented toward the Sun) near $(X, Y)=(-4,-3) \mathrm{kpc}$, and another cluster with clockwise fields near $(3,5) \mathrm{kpc}$.

\subsection{Rotation Measure Comparison}

The structure of the Galactic magnetic field can be studied with rotation measures of Galactic pulsars and extragalactic sources. We find that toward the second and third Galactic quadrants, which contain only six sources, all fields are oriented in the clockwise direction, while the nearest six sources in the first and fourth quadrants are consistent with a counterclockwise field. This agrees with rotation measure studies of the outer Galaxy, which also find an overall clockwise field (Lyne \& Smith 1989; Brown \& Taylor 2001). This also agrees with a reversal inward claimed in other papers from pulsar rotation measure estimates (Simard-Normandin \& Kronberg 1979; Rand \& Kulkarni 1989; Clegg et al. 1992).

In order to compare our results with the Galactic magnetic field deduced from rotation measures, we generated simulated rotation measure data from the line-of-sight magnetic field direction of our $\mathrm{OH}$ maser data. Our data consist of measurements of milligauss fields in situ around massive SFRs, while extragalactic rotation measures are line-of-sight integrals predominantly through the microgauss fields of the interstellar medium. We calculated simulated rotation measures along rays of Galactic longitude within the Galactic plane using the line-of-sight field directions from $\mathrm{OH}$ masers. The rotation measure $R M$ of radiation coming from a source at a distance $D$ is given by

$$
R M=8.1 \times 10^{5} \mathrm{rad} \mathrm{m}^{-2} \int_{D}^{0} n_{e} \mathbf{B} \cdot \mathbf{d l},
$$

where $n_{e}$ is the electron density in $\mathrm{cm}^{-3}, \mathbf{B}$ is the magnetic field in gauss, and $\mathbf{d l}$ is measured in parsecs. Typical values for these variables in the interstellar medium are $n_{e} \approx 0.03 \mathrm{~cm}^{-3}$ (Lyne, Manchester, \& Taylor 1985) and $B_{\|} \approx 2 \times 10^{-6} \mathrm{G}$ (Manchester 1974; Simard-Normandin \& Kronberg 1980; Heiles 1976). Given that magnetic fields implied by Zeeman splitting in massive SFRs exhibit some correlation on a scale of $\lesssim 2 \mathrm{kpc}$ (see $\S 5.2$ ), we postulated that the magnetic field around a massive SFR indicates the prevailing Galactic magnetic field direction in a sphere of radius $r$ centered at the massive SFR. We adopted $r \sim 0.5 \mathrm{kpc}$, which yields a contribution of 
about $50 \mathrm{rad} \mathrm{m}^{-2}$ to the rotation measure of a line of sight passing along the diameter of each sphere. Thus, we assigned a rotation measure of $\pm 50 \mathrm{rad} \mathrm{m}^{-2}$ to each sphere, depending only on the line-of-sight magnetic field direction in the massive SFR, and summed the rotation measures of the spheres intersected by the ray. For simplicity, each sphere was assumed to contribute $\pm 50 \mathrm{rad} \mathrm{m}^{-2}$ to the total rotation measure, regardless of the path length of the ray within the sphere. Note that since the radiation propagates from $D$ to $0, \mathbf{d l}$ is oriented toward the Sun, and $\mathbf{B} \cdot \mathbf{d l}$ is negative when the magnetic field is oriented away from the Sun. Thus, a positive magnetic field generates a negative rotation measure.

Our simulated rotation measures are plotted in Figure 4 for $r=0.5 \mathrm{kpc}$. Features of the plot are not highly sensitive to the value of $r$, but much lower values leave a large volume of the Galactic plane unsampled by our data, while much higher values result in a lot of overlap of the spheres. Superposed atop this plot are extragalactic rotation measures reproduced directly from Figure 9 of Rand \& Kulkarni (1989), based on data from Simard-Normandin, Kronberg, \& Button (1981). Our data agree reasonably well with the extragalactic rotation measure data. In the second and third Galactic quadrants, our data match the sign of the extragalactic rotation measures. While the extragalactic rotation measures are not generally of consistent sign in the first and fourth quadrants, agreement is found again at $l=15^{\circ}$, where the extragalactic rotation measures are decidedly positive. The simulated rotation measures we deduce from $\mathrm{OH}$ Zeeman splitting appear to fit the extragalactic data at least as well as, if not better than, the ring model of Rand \& Kulkarni (1989).

\subsection{Spiral Arms}

In many models, one would expect the Galactic field to be organized along spiral arms. However, investigating whether the Galactic field shows organization on a per-arm basis necessitates assignment of massive SFRs to spiral arms. Based on positions on both a plot of the Galaxy and on the longitude-velocity diagram in Figure 5, most of the sources in our study were assigned to a spiral arm, as listed in Table 4 . The above correlation analysis was repeated on the subset of sources associated with each spiral arm, and the results are included in Table 3. For the most part, no obvious correlations exist except for sources assigned to the Norma arm, where 8 of 10 sources (and all 7 in the fourth quadrant) have a magnetic field oriented clockwise with respect to the Galactic center.

As noted above, it is difficult to make strong statements pertaining to field directions in spiral arms. The shape and locations of the spiral arms in the Milky Way are still a matter of debate, and many of the source distances used in this study are kinematic and therefore subject to large enough errors that some could be placed in the wrong arms. Even assuming that the near/far kinematic distance ambiguity has been resolved correctly for all sources, a typical distance error by this method is about $1 \mathrm{kpc}$. Furthermore, the $\mathrm{OH}$ maser velocity may differ from the velocity of the massive SFR, which may in turn deviate quite significantly from uniform circular motion, 
as discussed in $\S 4.1$. Especially near $l=0^{\circ}$, where several spiral arms cross in a longitude-velocity diagram, it is difficult to reliably assign massive SFRs to spiral arms.

\subsection{Intra-source Field Reversals}

The problem of relating $\mathrm{OH}$ Zeeman magnetic field measurements to the Galactic magnetic field can be inverted. Assuming that there exists a large-scale Galactic field, do our results allow us to comment either on whether the magnetic field threading $\mathrm{OH}$ maser clumps are related to the (pre-collapse) local Galactic field or on whether intra-source field reversals confuse the issue too much to see conclusive evidence of large-scale field organization?

These are questions that cannot be answered conclusively with the VLA alone, due to its large beam size. For instance, no conclusive Zeeman pairs are found in the $1665 \mathrm{MHz}$ transition in the VLA data for W3(OH). In contrast, Bloemhof, Reid, \& Moran (1992) identified 23 Zeeman pairs in the same transition using VLBI. Significant blending of independent maser features within a single VLA beam often renders identification of Zeeman pairs ambiguous, as discussed in §4.5. When Zeeman pairs are identified with VLA resolution, they are often in short supply. We were able to find only one Zeeman pair in many of the massive SFRs shown in Table 1. One pair does not conclusively determine the prevailing magnetic field direction surrounding the region. Even in the simple case, depicted in Figure 6, of a conducting, spherical H II region in a uniform magnetic field, the line-of-sight projection of the magnetic field direction may vary depending on the inclination of the source (Bourke et al. 2001). A numerical simulation was run to estimate the fraction of sources whose line-of-sight magnetic field would be incorrectly inferred owing to projection effects for this simple geometry. Given an ensemble of ideal, spherical H II regions, each with a randomlyoriented prevailing magnetic field direction and a single magnetic field measurement taken locally at a random point on the projection of the sphere (as from a Zeeman pair), the line-of-sight direction of the measured field will be opposite to the line-of-sight direction of the prevailing magnetic field $25 \%$ of the time.

Intra-source field reversals have been seen across three of nine massive SFRs that were mapped in the main-line $\mathrm{OH}$ transitions with the VLBA: G351.775-0.538 (Argon, Reid, \& Menten 2002), W75N, and W75S (unpublished data). Thus, one-third of the population of massive SFRs mapped at milliarcsecond resolution show at least two distinct maser clumps with Zeeman pairs indicating opposite line-of-sight directions. While one might have expected that the magnetic field would be oriented randomly in a massive SFR owing to a complex evolutionary history that "scrambles" the pre-collapse field, massive SFRs with intra-source field reversals still exhibit an organized field structure. More likely, these reversals are a projection effect due to the geometry of the field lines relative to the observer. Still, the ionized sphere model is surely an oversimplification in some cases. For instance, the $\mathrm{OH}$ masers around W75N are aligned along two intersecting lines, one running roughly north-south and the other east-west (Baart et al. 1986). Our VLBA data show that the magnetic fields along the north-south line point consistently away from the Sun, while those in 
the east-west line point consistently toward the Sun. Baart et al. suggest that the masers trace expanding shock waves, while Slysh et al. (2002) claim to have found evidence that the north-south structure is a thin, rotating disk. Whatever the explanation of this source may be, the observed intra-source field reversal is clearly not a simple projection effect in a static environment.

\subsection{The Random Component of the Galactic Magnetic Field}

Even if the Galactic magnetic field consists of a large-scale field with regular structure, the turbulent field component may hinder clear detection of the pattern. Synchrotron polarization observations suggest that the ratio of field strengths of the regular to turbulent magnetic fields may

be between 0.6 and 1.0, with lower values more likely in spiral arms (Beck 2001). Assuming a uniformly random two-dimensional (in the Galactic plane) orientation of the turbulent component of the magnetic field, the line-of-sight orientation of the total field will be opposite to that of the regular component $8 \%$ to $20 \%$ of the time for the aforementioned range of field strength ratios. Thus, even if the pre-collapse magnetic field orientation is preserved at sites of $\mathrm{OH}$ maser emission, as many as one in five sources could imply a line-of-sight magnetic field opposite to the assumed underlying regular field. On the other hand, Brown \& Taylor (2001) present evidence that the random field may be partially aligned with the uniform field, rather than being isotropic. If this is indeed the case, the above estimates of the rate of discrepancy between the magnetic field direction implied by $\mathrm{OH}$ Zeeman splitting and the regular field direction may be overly pessimistic. Also, the random component of the Galactic magnetic field may become less significant between interstellar and GMC densities.

\section{Conclusions and Further Research}

We have found nearly 100 Zeeman pairs of ground-state OH masers using the data from Paper I. Combining these Zeeman pairs with others found in the literature, we have investigated the distribution in the Galactic plane of the line-of-sight orientation of magnetic fields in massive SFRs as deduced from $\mathrm{OH}$ maser Zeeman splitting. Our data do not support the original hypothesis of Davies (1974) that the large-scale magnetic field traced by $\mathrm{OH}$ masers is uniform in the clockwise sense, nor do we find that the inferred magnetic fields show a uniform direction in each spiral arm.

All six sources in the second and third Galactic quadrants, which are typically 1 or $2 \mathrm{kpc}$ distant, have a magnetic field oriented in the clockwise sense as viewed from the North Galactic Pole, while nearly all nearby sources in the first and fourth quadrants have a magnetic field oriented in the counterclockwise direction. This supports the hypothesis, deduced from pulsar rotation measures, that a reversal of the Galactic-scale magnetic field occurs near the galactocentric radius of the Sun.

Our method for identifying Zeeman pairs in VLA maps of OH masers is limited by the blending 
of maser components inevitable with a large $\left(1^{\prime \prime}-2^{\prime \prime}\right)$ beamsize. The higher resolution of VLBI allows many more maser spots to be detected and Zeeman pairs to be identified unambiguously. The increased detail in VLBI images also allows for the identification of intra-source magnetic field reversals, the interpretation of which may be important both for understanding the conditions of the magnetic field in massive SFRs and for obtaining enough data to rigorously test the hypothesis that the magnetic field deduced from $\mathrm{OH}$ maser Zeeman splitting in massive SFRs is correlated with the Galactic magnetic field. To this end, we are in the process of imaging interstellar $\mathrm{OH}$ masers with the VLBA. The results will be published in a future paper. 
Table 2. Pairs with no Splitting

\begin{tabular}{|c|c|c|c|c|c|c|c|c|c|c|}
\hline Source & Alias & $\begin{array}{l}\text { Dist. }^{a} \\
(\mathrm{kpc})\end{array}$ & $\begin{array}{l}\text { Freq. } \\
(\mathrm{MHz})\end{array}$ & $\begin{array}{r}S^{\mathrm{b}} \\
(\mathrm{Jy})\end{array}$ & $\begin{array}{c}\mathrm{LCP}- \\
v_{\mathrm{LSR}}^{\mathrm{b}} \\
\left(\mathrm{km} \mathrm{s}^{-1}\right)\end{array}$ & $\begin{array}{c}\Delta v^{\mathrm{b}} \\
\left(\mathrm{km} \mathrm{s}^{-1}\right)\end{array}$ & $\begin{array}{r}S^{\mathrm{b}} \\
(\mathrm{Jy})\end{array}$ & $\begin{array}{c}\mathrm{RCP} \\
v_{\mathrm{LSR}}^{\mathrm{b}} \\
\left(\mathrm{km} \mathrm{s}^{-1}\right)\end{array}$ & $\begin{array}{c}\Delta v^{\mathrm{b}} \\
\left(\mathrm{km} \mathrm{s}^{-1}\right)\end{array}$ & $\begin{array}{c}\Delta \theta^{\mathrm{c}} \\
(\operatorname{arcsec})\end{array}$ \\
\hline G002.143+0.010 & $\cdots$ & 7.5 & 1667 & 2.09 & 59.27 & 0.34 & 0.96 & 59.27 & 0.50 & 0.026 \\
\hline G009.622+0.195 & $\cdots$ & 5.7 & 1667 & 4.67 & 7.13 & 0.29 & 0.92 & 7.14 & 0.36 & 0.032 \\
\hline G043.167+0.010 & W49 N & 11.5 & 1665 & 10.05 & 7.88 & 0.36 & 32.08 & 7.88 & 0.43 & 0.014 \\
\hline G080.864+0.421 & $\cdots$ & 4.1 & 1665 & 20.10 & -8.46 & 0.26 & 26.02 & -8.46 & 0.24 & 0.009 \\
\hline G081.721+0.571 & W75 S & 2.0 & 1667 & 0.74 & -1.12 & 0.27 & 1.56 & -1.14 & 0.37 & 0.070 \\
\hline \multirow[t]{2}{*}{ G081.871+0.781 } & W75 N & 2.0 & 1665 & 26.00 & 3.09 & 0.35 & 29.77 & 3.07 & 0.36 & 0.002 \\
\hline & & & & 13.95 & 0.63 & 0.39 & 17.31 & 0.64 & 0.39 & 0.003 \\
\hline G111.533+0.757 & NGC 7538 & 2.8 & 1665 & 0.94 & -57.69 & 0.30 & 0.64 & -57.70 & 0.35 & 0.011 \\
\hline G133.946+1.064 & W3 OH & 2.2 & 1665 & 32.94 & -47.45 & 0.22 & 20.61 & -47.45 & 0.22 & 0.015 \\
\hline G350.011-1.341 & $\cdots$ & 3.1 & 1667 & 2.25 & -23.74 & 0.51 & 2.60 & -23.78 & 0.50 & 0.016 \\
\hline \multirow[t]{2}{*}{ G351.775-0.538 } & $\cdots$ & 2.7 & 1665 & 40.53 & -9.24 & 0.25 & 37.35 & -9.23 & 0.27 & 0.027 \\
\hline & & & & 11.66 & 1.22 & 0.50 & 11.66 & 1.22 & 0.43 & 0.099 \\
\hline
\end{tabular}

${ }^{a}$ Kinematic distances assuming $R_{0}=8.5 \mathrm{kpc}$ and $\Theta_{0}=220 \mathrm{~km} \mathrm{~s}^{-1}$, unless note in text says otherwise.

${ }^{b}$ Peak flux density, center velocity, and line width (FHWM) of left- and right-circular polarized lines, respectively, as determined by Gaussian fit.

${ }^{\mathrm{c}}$ Angular offset between LCP and RCP lines. 
Table 3. Correlation Results

\begin{tabular}{rrrrrrrr}
\hline \hline \multirow{2}{*}{$\begin{array}{c}\text { Separation } \\
(\mathrm{kpc})\end{array}$} & \multirow{2}{*}{$\begin{array}{c}\text { Pairs of } \\
\text { Sources }\end{array}$} & \multicolumn{6}{c}{ Percentage of Trial Runs Less/More Correlated } \\
& Overall & Car-Sgr & Cru-Sct & Local & Norma & Perseus \\
\hline 0.5 & 43 & $41 / 46$ & $14 / 65$ & $87 / 7$ & $\ldots / \ldots$ & $25 / 25$ & $0 / 63$ \\
1.0 & 111 & $23 / 70$ & $56 / 31$ & $14 / 72$ & $13 / 38$ & $56 / 19$ & $0 / 63$ \\
2.0 & 324 & $82 / 15$ & $81 / 15$ & $76 / 19$ & $44 / 25$ & $91 / 4$ & $19 / 44$ \\
5.0 & 1201 & $52 / 45$ & $56 / 36$ & $66 / 28$ & $0 / 38$ & $95 / 2$ & $45 / 17$ \\
10.0 & 2197 & $37 / 59$ & $42 / 48$ & $37 / 33$ & $0 / 38$ & $89 / 2$ & $30 / 45$ \\
20.0 & 2664 & $63 / 36$ & $0 / 83$ & $37 / 33$ & $0 / 38$ & $89 / 2$ & $49 / 18$ \\
\hline
\end{tabular}

Note. - The figures represent the percentage of trial runs for which the correlation function evaluated on the random data were less and more correlated than the actual data. Results to any separation are cumulative; i.e., all pairs of sources with separation less than or equal to a given distance are included in the correlation function results to that distance. The total number of pairs of distinct sources with separation less than the figure in the first column is given in the second column. 
Table 4. Sources and Field Directions by Arm

\begin{tabular}{|c|c|c|c|c|}
\hline $\begin{array}{c}\text { Galactic } \\
\text { Longitude }\end{array}$ & $\begin{array}{l}\text { Galactic } \\
\text { Latitude }\end{array}$ & $\begin{array}{l}\text { Distance } \\
\quad(\mathrm{kpc})\end{array}$ & $\begin{array}{c}\text { Magnetic } \\
\text { Field }^{\mathrm{a}}\end{array}$ & References \\
\hline \multicolumn{5}{|c|}{ Carina-Sagittarius } \\
\hline 17.639 & +0.155 & 2.1 & - & 1 \\
\hline 20.081 & -0.135 & 12.3 & + & 1,7 \\
\hline 32.744 & -0.076 & 2.3 & - & 1,2 \\
\hline 34.258 & +0.153 & 3.8 & + & $1,2,3$ \\
\hline 35.024 & +0.350 & 3.1 & + & 1,2 \\
\hline 35.197 & -0.743 & 2.2 & + & 1 \\
\hline 35.577 & -0.029 & 10.5 & - & 1 \\
\hline 43.796 & -0.127 & 3.0 & + & 1,2 \\
\hline 45.071 & +0.134 & 4.7 & + & $1,4,5$ \\
\hline 45.122 & +0.133 & 6.0 & - & $1,2,4$ \\
\hline 45.465 & +0.060 & 6.0 & + & 1 \\
\hline 49.469 & -0.370 & 6.0 & + & 1,6 \\
\hline 49.488 & -0.387 & 5.5 & + & $1,2,6$ \\
\hline 285.26 & -0.05 & 5.7 & - & 2 \\
\hline 294.51 & -1.62 & 1.4 & - & 2 \\
\hline 311.64 & -0.38 & 14.1 & - & 2 \\
\hline 344.581 & -0.022 & 2.0 & - & 1 \\
\hline 345.505 & +0.347 & 2.1 & - & 1,2 \\
\hline 345.699 & -0.090 & 1.6 & - & 1,2 \\
\hline 348.549 & -0.978 & 2.1 & + & $1,2,7$ \\
\hline 351.161 & +0.697 & 2.3 & - & 1 \\
\hline 351.416 & +0.646 & 2.0 & + & $1,2,7$ \\
\hline \multicolumn{5}{|c|}{ Crux-Scutum } \\
\hline 5.886 & -0.393 & 3.8 & + & 1 \\
\hline 11.03 & +0.06 & 3.2 & - & 2 \\
\hline 12.216 & -0.117 & 3.3 & - & 1 \\
\hline 12.680 & -0.181 & 4.0 & - & 1,8 \\
\hline 12.908 & -0.259 & 4.0 & + & 1 \\
\hline 28.199 & -0.048 & 5.7 & + & 1,3 \\
\hline 30.70 & -0.06 & 5.5 & + & 7 \\
\hline 323.459 & -0.079 & 4.1 & - & 2,10 \\
\hline 329.41 & -0.46 & 4.3 & - & 2 \\
\hline 331.34 & -0.34 & 4.7 & - & 7 \\
\hline 333.60 & -0.21 & 3.5 & + & 2 \\
\hline 339.62 & -0.12 & 2.8 & + & 2 \\
\hline 341.219 & -0.212 & 3.2 & - & 1 \\
\hline 343.128 & -0.063 & 3.1 & - & 1 \\
\hline 345.003 & -0.224 & 2.9 & - & $1,2,7$ \\
\hline 350.011 & -1.341 & 3.1 & - & 1 \\
\hline 359.138 & +0.032 & 3.1 & + & 1 \\
\hline \multicolumn{5}{|c|}{ Local } \\
\hline 69.540 & -0.976 & 1.3 & - & $1,3,9,11$ \\
\hline 81.77 & +0.60 & 2.0 & - & 3 \\
\hline 106.80 & +5.31 & 1.4 & + & 3 \\
\hline 109.871 & +2.114 & 0.7 & + & $1,3,12$ \\
\hline 213.706 & -12.60 & 0.9 & + & 1 \\
\hline
\end{tabular}




\section{REFERENCES}

Argon, A. L., Reid, M. J., \& Menten, K. M. 2000, ApJS, 129, 159 (Paper I)

Argon, A. L., Reid, M. J., \& Menten, K. M. 2002, in IAU Symposium 206, Cosmic MASERs: From Protostars to Blackholes, ed. V. Migenes and M. J. Reid (San Francisco: ASP), 367

Baart, E. E., Cohen, R. J., Davies, R. D., Rowland, P. R., \& Norris, R. P. 1986, MNRAS, 219, 145

Baart, E. E., \& Cohen, R. J. 1985, MNRAS, 213, 641

Baudry, A., Desmurs, J. F., Wilson, T. L., \& Cohen, R. J. 1997, A\&A, 325, 255

Beck, R. 2001, Space Sci. Rev., 99, 243

Bloemhof, E. E., Reid, M. J., \& Moran, J. M. 1992, ApJ, 397, 500

Brown, J. C., \& Taylor, A. R. 2001, ApJ, 563, L31

Bourke, T. L., Myers, P. C., Robinson, G., \& Hyland, A. R. 2001, ApJ, 554, 916

Campbell, B., \& Thompson, R. I. 1984, ApJ, 279, 650

Caswell, J. L. 2001, MNRAS, 326, 805

Caswell, J. R., \& Reynolds, J. E. 2001, MNRAS, 325, 1346

Caswell, J. L., \& Vaile, R. A. 1995, MNRAS, 273, 328

Clegg, A. W., Cordes, J. M., Simonetti, J. M., \& Kulkarni, S. R. 1992, ApJ, 386, 143

Cohen, R. J., Brebner, G. C., \& Potter, M. M. 1990, MNRAS, 246, 3P

Davies, R. D. 1974, in IAU Symposium 60, Galactic Radio Astronomy, ed. F. J. Kerr and S. C. Simonson III (Dordrecht:Reidel), p. 275

De Pree, C. G., Mehringer, D. M., \& Goss, W. M. 1997, ApJ, 482, 307

Desmurs, J. F., \& Baudry, A. 1998, A\&A, 340, 521

Dickel, J. R., Dickel, H. R., \& Wilson, W. J. 1978, ApJ, 223, 840

Fish, V. L., Reid, M. J., Argon, A. L., \& Menten, K. M. 2002, in IAU Symposium 206, Cosmic MASERs: From Protostars to Blackholes, ed. V. Migenes and M. J. Reid (San Francisco: ASP), 371

Fish, V. L., Reid., M. J., Wilner, D. J., \& Churchwell, E. 2003, ApJ, 587, 701

Forster, J. R., \& Caswell, J. L. 1989, A\&A, 213, 339 
Table 4-Continued

\begin{tabular}{|c|c|c|c|c|}
\hline $\begin{array}{l}\text { Galactic } \\
\text { Longitude }\end{array}$ & $\begin{array}{l}\text { Galactic } \\
\text { Latitude }\end{array}$ & $\begin{array}{l}\text { Distance } \\
(\mathrm{kpc})\end{array}$ & $\begin{array}{c}\text { Magnetic } \\
\text { Field }^{\mathrm{a}}\end{array}$ & References \\
\hline 8.67 & -0.36 & 4.8 & + & 2 \\
\hline 9.622 & +0.195 & 5.7 & - & 1 \\
\hline 10.624 & -0.385 & 4.8 & - & 1 \\
\hline 330.95 & -0.18 & 5.7 & + & 2,7 \\
\hline 336.36 & -0.14 & 10.3 & + & 2 \\
\hline 336.83 & +0.02 & 10.3 & + & 2 \\
\hline 337.61 & -0.06 & 12.8 & + & 2 \\
\hline 337.71 & -0.05 & 12.2 & + & 2,7 \\
\hline 344.42 & +0.05 & 13.4 & + & 2 \\
\hline 350.11 & +0.09 & 5.8 & + & 2 \\
\hline \multicolumn{5}{|c|}{ Perseus } \\
\hline 43.148 & +0.015 & 11.5 & + & 1,2 \\
\hline 43.165 & -0.028 & 11.5 & + & 1 \\
\hline 43.167 & +0.010 & 11.5 & - & 7 \\
\hline 70.293 & +1.601 & 8.3 & - & $1,3,11$ \\
\hline 75.782 & +0.343 & 5.0 & + & 11 \\
\hline 80.87 & +0.42 & 4.8 & - & 3 \\
\hline 111.533 & +0.757 & 2.8 & + & 1 \\
\hline 133.946 & +1.064 & 2.2 & + & $1,3,7,13$ \\
\hline 196.454 & -1.677 & 2.8 & + & 11 \\
\hline \multicolumn{5}{|c|}{ Unassigned $^{\mathrm{b}}$} \\
\hline 0.547 & -0.852 & 7.3 & - & 1 \\
\hline 0.666 & -0.034 & 8.2 & + & 1 \\
\hline 0.670 & -0.058 & 8.2 & - & 1 \\
\hline 0.672 & -0.031 & 8.2 & + & 1 \\
\hline 40.42 & +0.70 & 1.2 & - & 1,2 \\
\hline 300.97 & +1.15 & 4.4 & + & 2,7 \\
\hline 305.81 & -0.24 & 3.2 & - & 7 \\
\hline 347.628 & +0.149 & 9.8 & + & 1,2 \\
\hline 354.73 & +0.29 & 8.5 & - & 2 \\
\hline 355.345 & +0.146 & 23.1 & + & 1 \\
\hline 359.436 & -0.103 & 8.2 & + & 1 \\
\hline
\end{tabular}

${ }^{a}$ Clockwise (+) or counterclockwise (-) magnetic field, as viewed from the North Galactic Pole. Note that "positive" magnetic fields (pointing away from the Sun) are clockwise in the first and second Galactic quadrants but counterclockwise in the third and fourth quadrants.

${ }^{b}$ Includes sources in the Galactic center.

References. - (1) this paper; (2) Caswell \& Vaile 1995; (3) Baudry et al. 1997; (4) Baart \& Cohen 1985; (5) Zheng 1997; (6) Gaume \& Mutel 1987; (7) Reid \& Silverstein 1990; (8) Zheng 1991; (9) Desmurs \& Baudry 1998; (10) Caswell \& Reynolds 2001; (11) in preparation; (12) Cohen, Brebner, \& Potter 1990; (13) García-Barreto et al. 1988 
García-Barreto, J. A., Burke, B. F., Reid, M. J., Moran, J. M., Haschick, A. D., \& Schilizzi, R. T. 1988, ApJ, 326, 954

Gaume, R. A., \& Mutel, R. L. 1987, ApJS, 65, 193

Han, J. L., Manchester, R. N., Lyne, A. G., \& Qiao, G. J. 2002, ApJ, 570, L17

Haschick, A. D., \& Ho, P. T. P. 1983, ApJ, 267, 638

Heiles, C. 1976, ARA\&A, 14, 1

Hofner, P., Kurtz, S., Churchwell, E., Walmsley, C. M., \& Cesaroni, R. 1994, ApJ, 429, L85

Humphreys, R. M., 1978, ApJS, 38, 309

Lyne, A. G., Manchester, R. N., \& Taylor, J. H. 1985, MNRAS, 213, 613

Lyne, A. G., \& Smith, F. G. 1989, MNRAS, 237, 533

Manchester, R. N. 1974, ApJ, 188, 637

Mouschovias, T. C. 1976, ApJ, 207, 141

Rand, R. J., \& Kulkarni, S. R. 1989, ApJ, 343, 760

Rand, R. J., \& Lyne, A. G. 1994, MNRAS, 268, 497

Reid, M. J., Haschick, A. D., Burke, B. F., Moran, J. M., Johnston, K. J., \& Swenson, G. W., Jr. 1980, ApJ, 239, 89

Reid, M. J., Readhead, A. C. S., Vermeulen, R. C., \& Treuhaft, R. N. 1999, ApJ, 524, 816

Reid, M. J., \& Moran, J. M. 1981, ARA\&A, 19, 231

Reid, M. J., Myers, P. C., \& Bieging, J. H. 1987, ApJ, 312, 830

Reid, M. J., \& Silverstein, E. M. 1990, ApJ, 361, 483

Scoville, N. Z., Yun, M. S., Clemens, D. P., Sanders, D. B., \& Waller, W. H. 1987, ApJS, 63, 821

Simard-Normandin, M., \& Kronberg, P. P. 1979, Nature, 279, 115

Simard-Normandin, M., \& Kronberg, P. P. 1980, ApJ, 242, 74

Simard-Normandin, M., Kronberg, P. P., \& Button, S. 1981, ApJS, 45, 97

Slysh, V. I., Migenes, V., Val'tts, I. E., Lyubchenko, S. Yu., Horiuchi, S., Altunin, V. I., Fomalont, E. B., \& Inoue, M. 2002, ApJ, 564, 317

Sofue, Y., Klein, U., Beck, R., \& Wielebinski, R. 1986, Ap\&SS, 119, 191 
Taylor, J. H., \& Cordes, J. M. 1993, ApJ, 411, 674

Wilson, T. L. 1974, A\&A 31, 83

Zheng, X. 1991, ChA\&A, 15, 219

Zheng, X. 1997, ChA\&A, 21, 182

Zheng, X., Reid, M. J., \& Moran, J. M. 2000, A\&A, 357, L37 


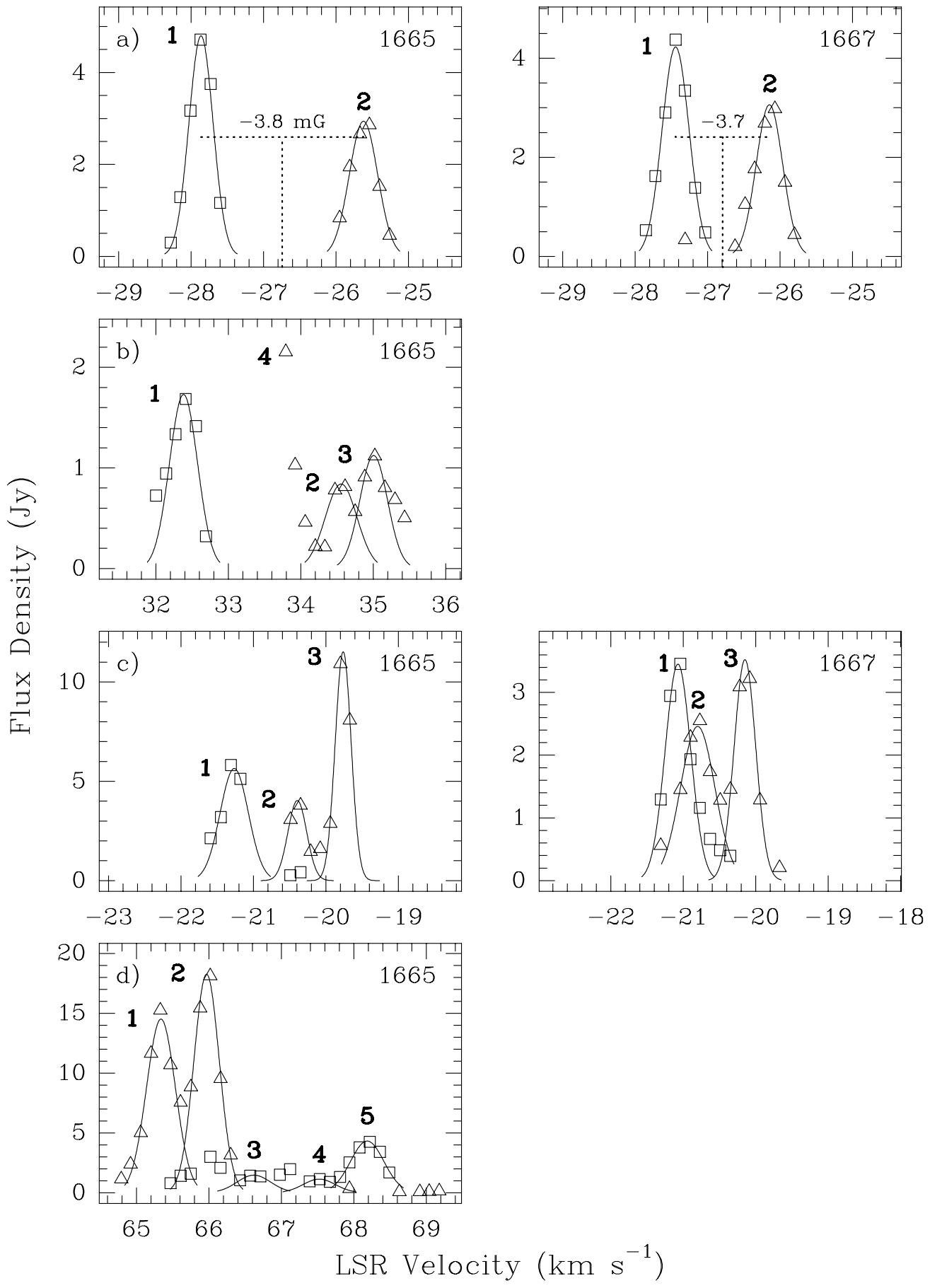

Fig. 1. - Sample spectra of windows used to find Zeeman pairs. Squares and triangles mark RCP and LCP emission, respectively. Gaussian fits to the lines are shown, and lines and features are numbered for reference. The number in the upper right corner marks the transition frequency, in MHz. (a-d) Windows in G351.775-0.538, G032.744-0.076, K3-50, and G045.465+0.047. The 1665 and $1667 \mathrm{MHz}$ windows in (a) and (c) are centered at approximately the same position. The magnetic field and center velocity deduced from Zeeman splitting are shown in (a). 


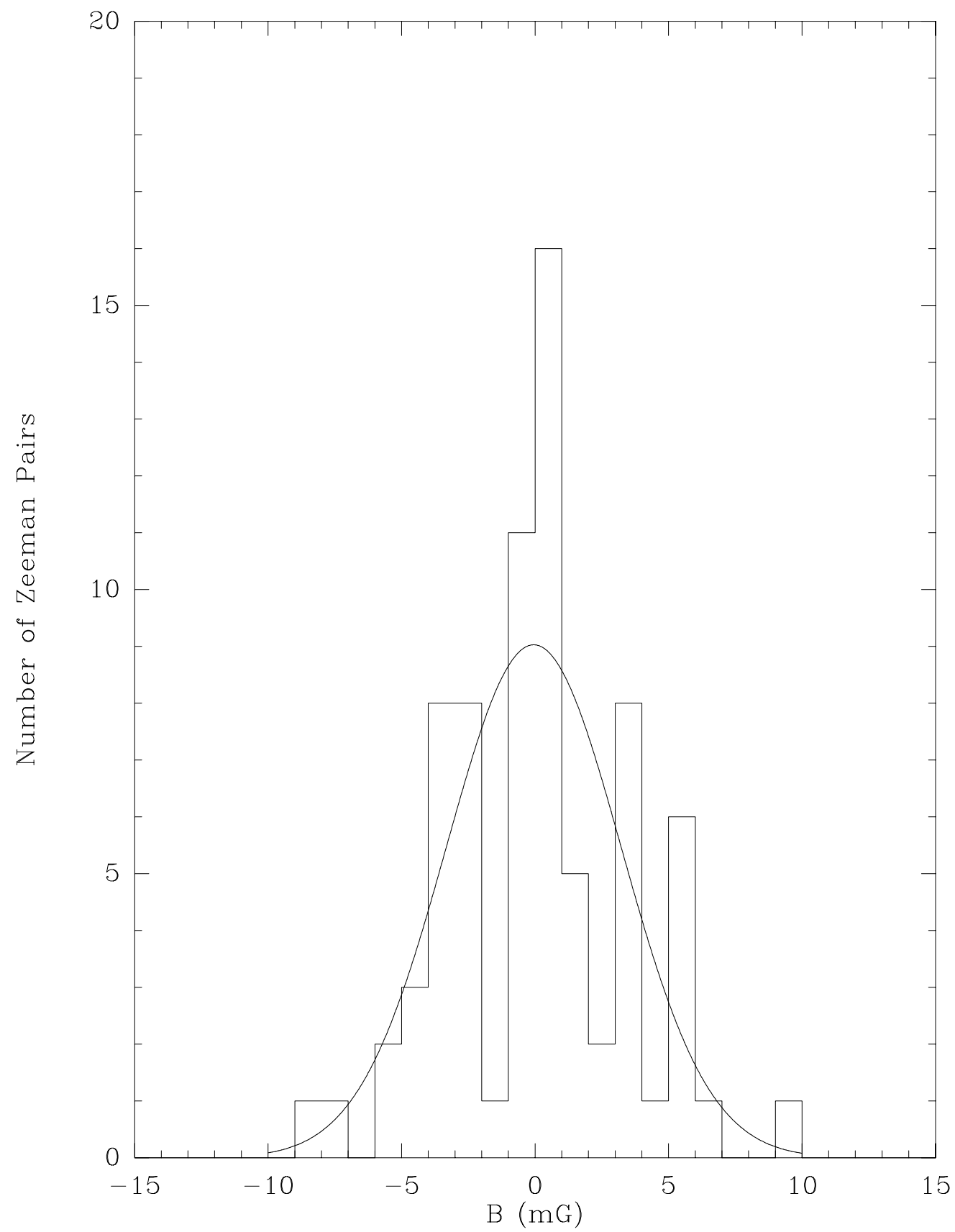

Fig. 2.- Histogram of the number of Zeeman pairs found with each magnetic field strength, binned by $1 \mathrm{mG}$. Positive (negative) field strengths represent magnetic fields oriented in the hemisphere away from (toward) the Sun. A best-fit Gaussian is also shown. The $-25.4 \mathrm{mG}$ pair in G081.871+0.781 is excluded for reasons outlined in $\S 4.3$. 


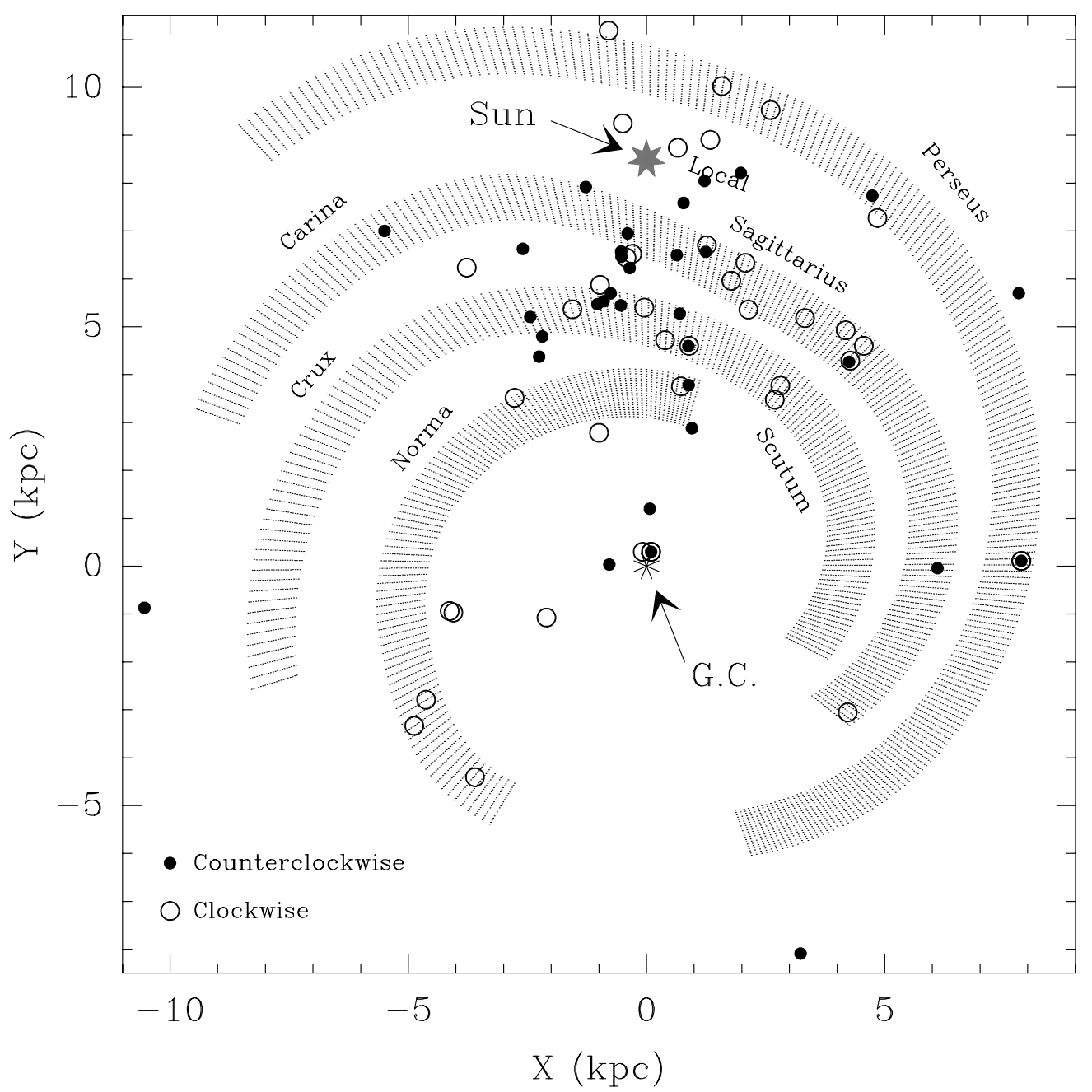

Fig. 3.- Line-of-sight magnetic field directions deduced from $\mathrm{OH}$ maser Zeeman splitting. Seventyfour star-forming regions are plotted, distinguishing 41 with an overall magnetic field oriented in a clockwise sense from 33 with field oriented counterclockwise as viewed from above the Galactic center (i.e., North Galactic Pole). The locations of the Sun and Galactic center are marked. The spiral arms of Taylor \& Cordes (1993) are indicated with shading and labelled by their common names. 


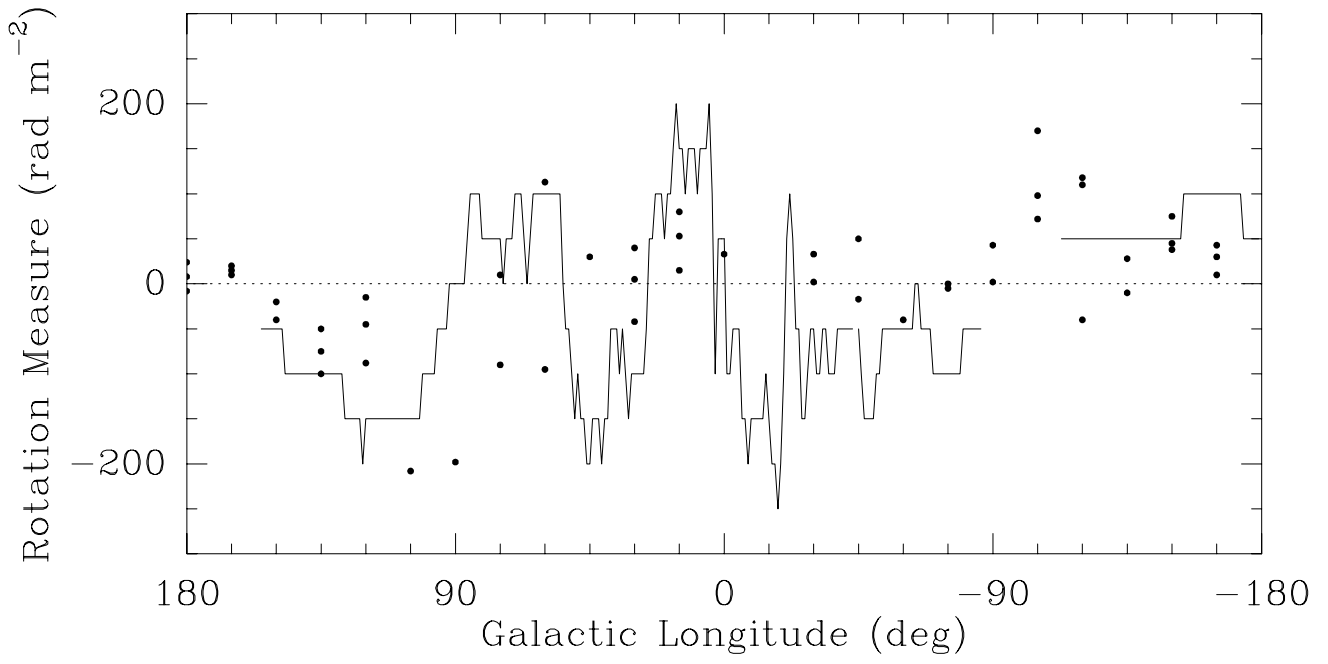

Fig. 4.- Plot of simulated rotation measures generated from magnetic field directions deduced from $\mathrm{OH}$ Zeeman splitting. No simulated rotation measures are plotted for Galactic longitudes unsampled by the data of Figures 3 and 5 . The dots represent extragalactic rotation measure data as taken from Rand \& Kulkarni (1989) at Galactic latitude $b=-15,0$, and $15^{\circ}$. See $\S 5.3$ for further details. 


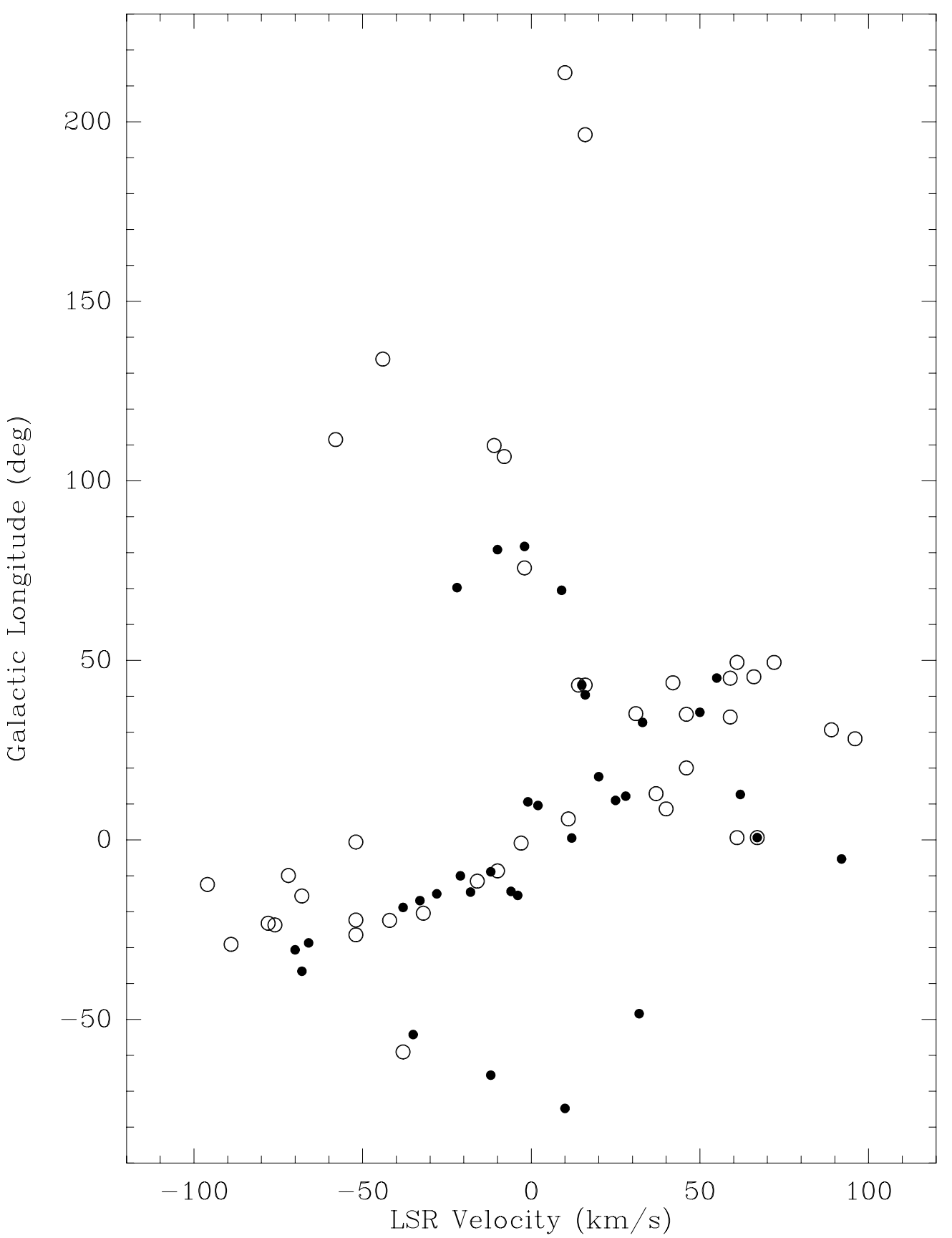

Fig. 5.- Longitude-velocity diagram of magnetic field directions deduced from $\mathrm{OH}$ maser Zeeman splitting. Open and filled circles are defined as in Figure 3. 


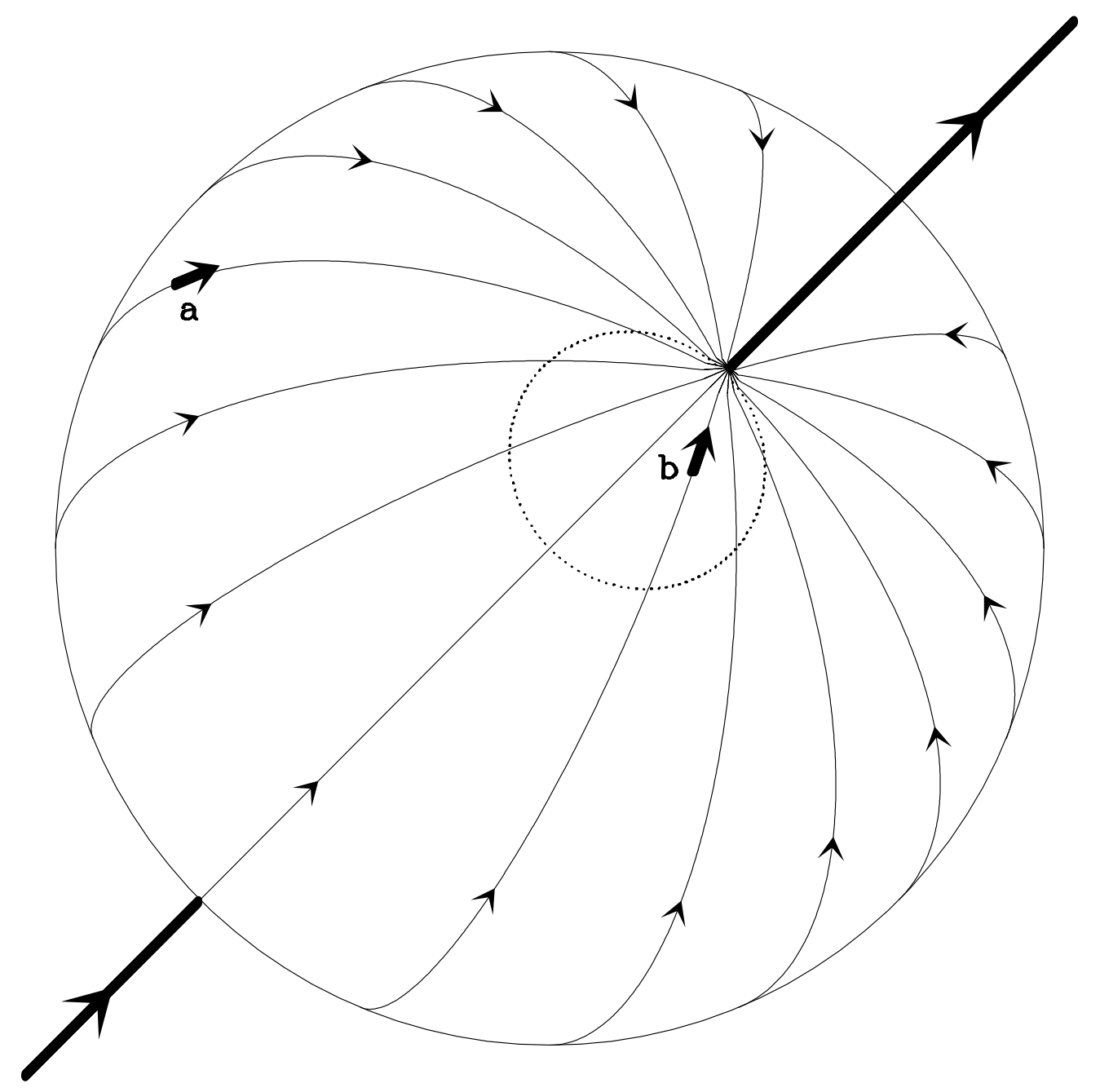

Fig. 6.- Spherical bubble model of an H II region in a magnetic field. The field is oriented to the upper right and out of the page, inclined $30^{\circ}$ to the line of sight. The arrows at $\mathbf{a}$ and $\mathbf{b}$ represent the local magnetic field at two points as might be deduced from Zeeman splitting. The line-of-sight projection of the magnetic field is out of the page outside the dotted curve (as at a) and into the page inside the curve (as at b). In the limiting cases of the magnetic field aligned along (perpendicular to) the line of sight, the line-of-sight direction of the field is reversed over none (half) of the projected area of the sphere. For a uniform distribution of magnetic field directions intersecting a sphere at all possible angles, on average the line-of-sight direction of the magnetic field is reversed over $25 \%$ of the area of the projected sphere. 